\title{
UMA PROPRIEDADE ESTRUTURAL DO PROBLEMA DE PROGRAMAÇÃO DA PRODUÇÃO FLOW SHOP PERMUTACIONAL COM TEMPOS DE SETUP
}

\author{
João Vitor Moccellin * \\ Departamento de Engenharia de Produção \\ Escola de Engenharia de São Carlos \\ Universidade de São Paulo (USP) \\ São Carlos - SP \\ jvmoccel@sc.usp.br \\ Marcelo Seido Nagano \\ Departamento de Engenharia de Produção \\ Escola de Engenharia de São Carlos \\ Universidade de São Paulo (USP) \\ São Carlos - SP \\ drnagano@usp.br \\ * Corresponding author / autor para quem as correspondências devem ser encaminhadas \\ Recebido em 08/2006; aceito em 10/2007 após 1 revisão \\ Received August 2006; accepted October 2007 after one revision
}

\begin{abstract}
Resumo
Neste artigo apresenta-se uma propriedade estrutural do problema de programação da produção flow shop permutacional com tempos de setup das máquinas separados dos tempos de processamento das tarefas, a qual foi identificada a partir de investigações que foram realizadas sobre as características do problema. Tal propriedade fornece um limitante superior do tempo de máquina parada entre a sua preparação e o início de execução das tarefas. Utilizando a propriedade, o problema original de programação da produção com minimização do makespan pode ser resolvido de maneira heurística por meio de uma analogia com o problema assimétrico do caixeiro-viajante.
\end{abstract}

Palavras-chave: programação da produção; flow shop permutacional; tempos de setup.

\begin{abstract}
This paper deals with the permutation flow shop scheduling problem with separated machine setup times. As a result of an investigation on the problem characteristics a structural property is introduced. Such a property provides an upper bound on the idle time of the machines between the setup task and the job processing. As an application of this property, the original scheduling problem with the makespan criterion can be heuristically solved by an analogy with the asymmetric traveling salesman problem.
\end{abstract}

Keywords: production scheduling; permutation flow shop; setup times. 


\section{Introdução}

O problema de programação flow shop é um problema de programação da produção no qual $\mathrm{n}$ tarefas devem ser processadas, na mesma seqüência, em um conjunto de m máquinas distintas. Um caso específico de programação flow shop, denominado permutacional, é quando em cada máquina mantém-se a mesma ordem de processamento das tarefas.

A solução do problema consiste em determinar dentre as (n!) seqüências possíveis das tarefas, aquela que otimiza alguma medida de desempenho da programação, sendo que as usuais consistem na minimização da Duração Total da Programação (makespan), ou minimização do Tempo Médio de Fluxo. A primeira relaciona-se a uma utilização eficiente dos recursos (máquinas) enquanto que a segunda busca minimizar o estoque em processamento.

Esse problema de programação da produção tem sido intensamente estudado na literatura, desde os resultados reportados por Johnson (1954) para o problema com somente duas máquinas. Hejazi \& Saghafian (2005) efetuaram uma ampla revisão da literatura referente ao ambiente de produção flow shop, mostrando que a maioria das pesquisas efetuadas considera os tempos de preparação (setup) das máquinas não significativos ou então os incluem nos tempos de processamento das tarefas. Isto simplifica a análise das aplicações, porém afeta a qualidade da programação quando tais tempos têm uma variabilidade relevante em função da ordenação das tarefas nas máquinas ou mesmo quando as máquinas podem ser preparadas antecipadamente para executarem as tarefas, não necessitando aguardar o término das tarefas nas máquinas precedentes.

Recentemente, o European Journal of Operational Research editou um número especial em comemoração aos 50 anos de publicação do primeiro artigo sobre programação da produção flow shop (Johnson, 1954). No Editorial paper dessa edição, Gupta \& Stafford Jr. (2006) procuram mostrar a evolução dos problemas e métodos de solução em ambiente flow shop nas últimas 5 décadas. Nesse contexto, pode-se observar que a consideração de problemas com um tratamento explícito dos tempos de preparação das máquinas praticamente teve início a partir da terceira década (1975-1984), com um aumento progressivo de interesse por parte dos pesquisadores a partir da quarta década. Essa tendência de intensificação de esforços em pesquisas abordando tais problemas encontra-se detalhada na revisão da literatura efetuada por Cheng, Gupta \& Wang (2000). Nesse artigo, os autores apresentam uma classificação dos problemas de programação da produção flow shop com tempos de setup e um conjunto de trabalhos relevantes realizados. Na conclusão do artigo são identificados alguns temas com potencial para futuras pesquisas, entre os quais o problema com múltiplos estágios (número de máquinas $\mathrm{m}>2$ ), sistemas de produção no-wait e modelos com medidas de desempenho multi-critério.

A consideração explícita dos tempos de preparação das máquinas é necessária em sistemas de produção como indústrias químicas, por exemplo, na produção de tintas onde o processo de limpeza é diferenciado dependendo da cor que estava sendo produzida e daquela que será fabricada em seguida (Simons Jr., 1992). Tal ambiente de produção também é comum em indústrias gráficas, têxteis e de produtos plásticos (Das, Gupta \& Khumawala, 1995).

Basicamente, existem dois tipos de tempos de preparação de máquinas tratados separadamente dos tempos de processamento das tarefas. No primeiro, tal tempo depende somente da tarefa a ser executada (independente da seqüência). O segundo depende tanto da tarefa a ser executada quanto daquela que foi processada imediatamente antes na mesma máquina, portanto dependente da seqüência. 
Este trabalho trata do problema flow shop permutacional com tempos de preparação das máquinas separados dos tempos de processamento das tarefas, podendo ser dependentes ou não da seqüência de execução das tarefas nas máquinas, que será denotado por FSP-TPS, e tem como objetivo apresentar uma propriedade relevante desse problema de programação da produção, proveniente de um estudo na sua estrutura. Na literatura, esse problema é considerado fortemente $N P$-hard. Essa complexidade pode ser entendida por meio da breve exposição que segue.

Garey, Johnson \& Sethi (1976) provaram que quando a medida de desempenho for o tempo médio de fluxo, o problema clássico de programação da produção flow shop permutacional, com os tempos de setup incluídos nos tempos de processamento das tarefas, é fortemente NP-hard para um ambiente com somente 2 máquinas. A mesma complexidade ocorre na minimização da duração total da programação (makespan) para o ambiente de produção com 3 máquinas. Conclui-se, portanto, que para o caso geral com um número de máquinas maior que dois tais problemas também são fortemente NP-hard. Pinedo (1995) apresenta uma estrutura hierárquica de complexidade envolvendo as denominadas Medidas de Desempenho Regulares, duas das quais são as mencionadas acima (tempo médio de fluxo e makespan). Na referida estrutura, essas duas medidas de desempenho ocupam os níveis de menor complexidade, o que permite concluir que a complexidade fortemente NP-hard é mantida para qualquer medida de desempenho regular. Para finalizar, a consideração explícita dos tempos de setup, separados dos tempos de processamento das tarefas, leva o problema a um nível superior de complexidade (Pinedo, 1995). Portanto, pode-se concluir que o problema FSP-TPS, tratado neste trabalho, é fortemente NP-hard.

\subsection{Problema FSP-TPS com tempos de preparação independentes da seqüência das tarefas}

O exame da literatura indica que o primeiro trabalho para este caso do problema FSP-TPS foi desenvolvido por Yoshida \& Hitomi (1979) para um flow shop com apenas duas máquinas, com o objetivo de minimizar o makespan. Os autores mostraram que o problema pode ser resolvido com solução ótima, aplicando-se a tradicional regra de Johnson (1954). Diversos outros trabalhos, com minimização do makespan e solução ótima, surgiram posteriormente para o mesmo caso de duas máquinas, porém com restrições adicionais como due dates para as tarefas (Khurana \& Bagga, 1985), tempos de remoção das tarefas (removal times) e condição de processamento contínuo (Gupta, Strusevich \& Zwaneveld, 1997). Outros trabalhos, também buscando a solução ótima, utilizaram medidas de desempenho da programação tais como a minimização do tempo médio de fluxo (Bagga \& Khurana, 1986), e a minimização do lateness máximo (Allahverdi \& Aldowaisan, 1998).

Para flow shops com múltiplas máquinas, no trabalho já mencionado de Yoshida \& Hitomi (1979) foi mostrado que mesmo para três máquinas, a minimização do makespan não necessariamente é obtida por uma programação permutacional. Mesmo assim, para o caso de diversas máquinas, as pesquisas relatadas na literatura consideram somente soluções permutacionais obtidas por métodos heurísticos. Nessas pesquisas, busca-se a minimização do makespan sob restrições como, por exemplo, buffer limitado (Park \& Steudel, 1991), tempos de transferência das tarefas entre as máquinas (Cao \& Bedworth, 1992) e tempos de remoção das tarefas não nulos (Rajendran \& Ziegler, 1997). 


\subsection{Problema FSP-TPS com tempos de preparação dependentes da seqüência das tarefas}

Para este caso de tempos de preparação das máquinas dependentes da seqüência das tarefas, o procedimento pioneiro de solução do problema foi desenvolvido por Gupta $(1969,1975)$ fundamentado na técnica de busca lexicográfica.

Para flow shops com duas máquinas, Corwin \& Esogbue (1974) propuseram um procedimento de Programação Dinâmica para obtenção da solução ótima, quanto ao makespan, porém com tempos de preparação dependentes da seqüência das tarefas em somente uma das máquinas. Os primeiros métodos heurísticos para o ambiente com duas máquinas foram propostos por Gupta \& Darrow (1985, 1986). Os métodos foram avaliados entre si considerando diferentes relações na ordem de grandeza dos tempos de processamento das tarefas e dos tempos de preparação das máquinas.

A grande maioria dos trabalhos reportados na literatura, para o caso de múltiplas máquinas, tem como medida de desempenho o makespan. Mesmo com aplicação restrita a problemas de pequeno porte, diversos métodos de solução ótima foram desenvolvidos, utilizando técnicas de Programação Linear Inteira Mista (Srikar \& Ghosh, 1986; Stafford \& Tseng, 1990; Rios-Mercado \& Bard, 1996) e métodos de enumeração Branch \& Bound (RiosMercado \& Bard, 1999a).

Para problemas de médio e grande porte salientam-se os métodos heurísticos. Os mais conhecidos são os métodos denominados SETUP e TOTAL propostos por Simons Jr. (1992) e um procedimento do tipo GRASP (Greedy Randomized Adaptive Search Procedure) desenvolvido por Rios-Mercado \& Bard (1998). Os métodos SETUP e TOTAL fundamentam-se em uma analogia do problema FSP-TPS com o problema assimétrico do caixeiro-viajante (ATSP - Asymmetric Traveling Salesman Problem). No caso do TOTAL, a matriz de "distâncias" entre pares de tarefas é obtida considerando-se a soma dos tempos de processamento das tarefas e dos tempos de preparação em todas as máquinas. No SETUP consideram-se somente os tempos de preparação das máquinas. No trabalho de RiosMercado \& Bard (1998) foi efetuada uma comparação de desempenho entre o método GRASP e o SETUP. De maneira geral, o GRASP superou o SETUP quando os tempos de preparação das máquinas foram gerados a partir de uma distribuição uniforme discreta no intervalo [1, 10] e os tempos de processamento das tarefas utilizando a distribuição [1, 99]. Entretanto, quando esta última distribuição foi utilizada para ambos, tempos de preparação e processamento, o método SETUP teve um desempenho melhor que o GRASP. Rios-Mercado \& Bard (1999b) propuseram uma extensão do método SETUP (Simons Jr., 1992) que foi denominado HYBRID. Essencialmente, esse método em uma primeira fase incorpora ao SETUP alguns aspectos característicos do problema FSP-TPS, e na segunda fase procura melhorar a solução inicial por meio de um procedimento de busca local. Os resultados advindos de uma experimentação computacional mostraram que, em geral, o HYBRID pode ser considerado superior ao método GRASP, quando os tempos de preparação das máquinas apresentam uma maior variabilidade, por exemplo, quando são gerados a partir de uma distribuição uniforme discreta no intervalo $[1,50]$ em comparação com os valores de uma distribuição $[1,10]$. 


\section{Uma Propriedade Estrutural do Problema FSP-TPS}

Considere-se o problema de Programação Flow Shop Permutacional, com $\mathrm{n}$ tarefas $\left\{\mathrm{J}_{1}, \mathrm{~J}_{2}, \ldots \mathrm{J}_{\mathrm{i}}, \ldots \mathrm{J}_{\mathrm{n}}\right\}$ e $\mathrm{m}$ máquinas $\left\{\mathrm{M}_{1}, \mathrm{M}_{2}, \ldots, \mathrm{M}_{\mathrm{k}}, \ldots, \mathrm{M}_{\mathrm{m}}\right\}$.

No problema tradicional, com os tempos de preparação das máquinas incluídos nos tempos de processamento das tarefas, são conhecidas as seguintes relações de viabilidade entre tempos de máquina parada e tempos de espera entre operações sucessivas de uma mesma tarefa, quando se considera que a tarefa $\mathrm{J}_{\mathrm{u}}$ precede diretamente $\mathrm{J}_{\mathrm{v}}$ na seqüência de execução das $n$ tarefas (French, 1982):

a) $X_{u \text { v }}^{\mathrm{k}}+\mathrm{p}_{\mathrm{k}, \mathrm{v}}+\mathrm{Y}_{\mathrm{u} \text { v }}^{\mathrm{k}}=\mathrm{Y}_{\mathrm{tu}}^{\mathrm{k}}+\mathrm{p}_{\mathrm{k}+1, \mathrm{u}}+\mathrm{X}_{\mathrm{uv}}^{\mathrm{k}+1}, \mathrm{e}$

b) Se $X_{u \text { v }}^{\mathrm{k}+1}>0$ então $Y_{u \text { v }}^{\mathrm{k}}=0$

Se $Y_{u \text { v }}^{\mathrm{k}}>0$ então $X_{u \text { v }}^{\mathrm{k}+1}=0$,

onde

$X_{i j}^{q}=$ intervalo de tempo entre o término de $J_{i}$ e o início de $J_{j}$ na máquina $M_{q}$;

$\mathrm{p}_{\mathrm{q}, \mathrm{j}}=$ tempo de processamento de $\mathrm{J}_{\mathrm{j}}$ na máquina $\mathrm{M}_{\mathrm{q}}$ (incluindo o tempo de preparação da máquina);

$\mathrm{Y}_{\mathrm{i} j}^{\mathrm{q}}=$ intervalo de tempo entre o término de $\mathrm{J}_{\mathrm{j}}$ na máquina $\mathrm{M}_{\mathrm{q}}$ e o seu início na máquina $\mathrm{M}_{\mathrm{q}+1}$, com $\mathrm{J}_{\mathrm{i}}$ precedendo diretamente $\mathrm{J}_{\mathrm{j}}$.

As relações (1) a (3) são ilustradas na Figura 1.

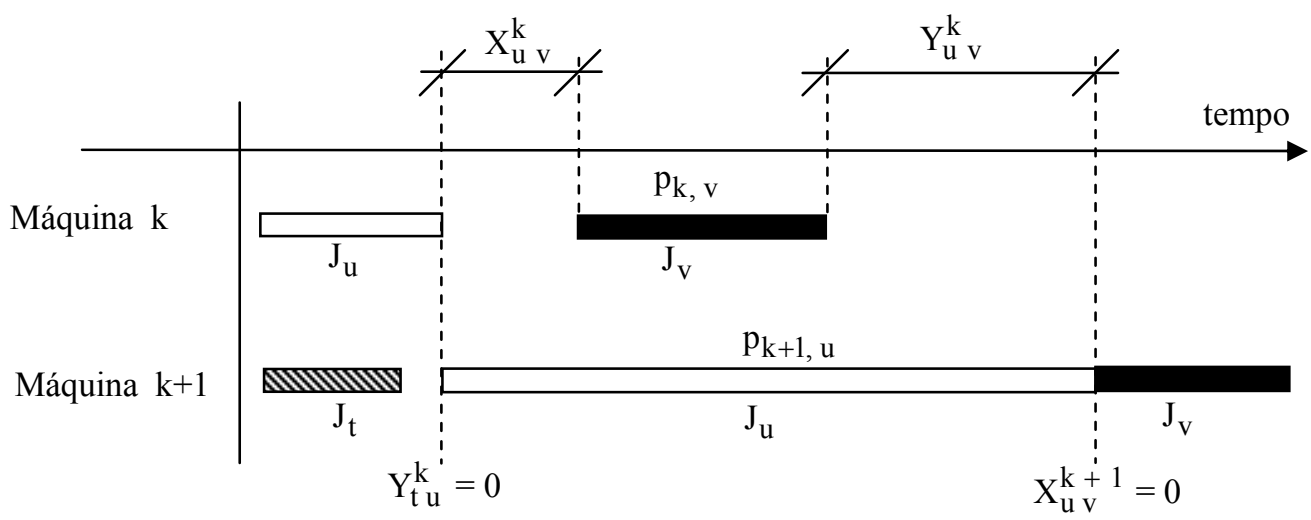

Figura 1 - Relações de viabilidade para duas tarefas adjacentes. 
Para o problema FSP-TPS, objeto deste trabalho, considere-se duas tarefas adjacentes quaisquer $\mathrm{J}_{\mathrm{u}}$ e $\mathrm{J}_{\mathrm{v}}$, e a atividade de preparação das máquinas nessa condição de tarefas sucessivas (independente ou não da seqüência), denotada por $S_{u v}$. Desta forma, $S_{u v}$ pode ser considerada como se fosse mais uma tarefa a ser sempre executada entre $J_{u}$ e $J_{v}$. Além disso, uma vez concluída a tarefa $\mathrm{J}_{\mathrm{u}}$ em uma máquina qualquer, essa máquina já pode ser preparada para executar a tarefa $\mathrm{J}_{\mathrm{v}}$, não necessitando aguardar o término da operação de $\mathrm{J}_{\mathrm{V}}$ na máquina anterior. Em algumas situações a preparação da máquina somente seria completada com a tarefa $\mathrm{J}_{\mathrm{v}}$ na máquina, por exemplo, para o posicionamento e fixação da peça (tarefa). Nesse caso, o tempo para tal atividade é incorporado ao tempo de processamento de $\mathrm{J}_{\mathrm{v}}$ na mencionada máquina. Portanto, não há tempo de máquina parada entre o término de $\mathrm{J}_{\mathrm{u}}$ e o início da preparação $\mathrm{S}_{\mathrm{uv}}$.

Assim, pode-se considerar que $\mathrm{J}_{\mathrm{u}}, \mathrm{S}_{\mathrm{uv}}$ e $\mathrm{J}_{\mathrm{v}}$ seriam três "tarefas" sucessivas em uma seqüência qualquer das tarefas. Desta forma, a expressão de viabilidade (1) entre tarefas sucessivas pode ser aplicada para os pares $\left(\mathrm{J}_{\mathrm{u}}, \mathrm{S}_{\mathrm{uv}}\right)$ e $\left(\mathrm{S}_{\mathrm{uv}}, \mathrm{J}_{\mathrm{v}}\right)$, levando a:

$$
\begin{aligned}
& \mathrm{X}_{\mathrm{uS} \mathrm{S}_{\mathrm{uv}}}^{\mathrm{k}}+\mathrm{s}_{\mathrm{k}, \mathrm{uv}}+\mathrm{Y}_{\mathrm{u} \mathrm{S}_{\mathrm{uv}}}^{\mathrm{k}}=\mathrm{Y}_{\mathrm{S}_{\mathrm{tu}}^{\mathrm{u}}}^{\mathrm{k}}+\mathrm{p}_{\mathrm{k}+1, \mathrm{u}}+\mathrm{X}_{\mathrm{u} \mathrm{S}_{\mathrm{uv}}^{\mathrm{k}+1}} \quad\left(\text { entre } \mathrm{J}_{\mathrm{u}} \text { e } \mathrm{S}_{\mathrm{uv}}\right)
\end{aligned}
$$

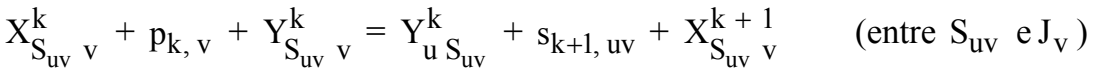

onde $\mathrm{s}_{\mathrm{q}}$, uv é o tempo de preparação da máquina $\mathrm{M}_{\mathrm{q}}$ para execução da tarefa $\mathrm{J}_{\mathrm{v}}$, que é diretamente precedida pela tarefa $J_{\mathrm{u}}$ na seqüência de execução das $n$ tarefas.

Na expressão (4) tem-se $\mathrm{X}_{\mathrm{u} \mathrm{S}}^{\mathrm{k}}=\mathrm{X}_{\mathrm{uv}}^{\mathrm{k}+1}=0$, o que leva a

$\mathrm{s}_{\mathrm{k}, \mathrm{uv}}+\mathrm{Y}_{\mathrm{u} \mathrm{S}}^{\mathrm{k}}=\mathrm{Y}_{\mathrm{S}_{\mathrm{tu}} \mathrm{u}}^{\mathrm{k}}+\mathrm{p}_{\mathrm{k}+1, \mathrm{u}}$, e portanto, $\mathrm{Y}_{\mathrm{u} \mathrm{S}}^{\mathrm{k}}=\mathrm{Y}_{\mathrm{S}_{\mathrm{tu}} \mathrm{u}}^{\mathrm{k}}+\mathrm{p}_{\mathrm{k}+1, \mathrm{u}}-\mathrm{s}_{\mathrm{k}, \mathrm{uv}}$.

Na expressão (6) é importante observar que $\mathrm{Y}_{\mathrm{u} \mathrm{S}_{\mathrm{uv}}}^{\mathrm{k}}$ não necessariamente mantém a condição de ser positivo ou nulo. Nesse caso, a data de término da preparação $\mathrm{S}_{\mathrm{uv}}$ na máquina $\mathrm{M}_{\mathrm{k}}$ é maior que a data de término dessa preparação na máquina $\mathrm{M}_{\mathrm{k}+1}$, conforme ilustra a Figura 2. Ou seja, não há restrição de precedência entre as atividades de preparação de máquinas sucessivas para execução de uma tarefa qualquer. É importante observar também que o fato de $\mathrm{Y}_{\mathrm{u} \mathrm{S}}^{\mathrm{k}}$ eventualmente ser negativo não elimina a validade das expressões (4) a (6). Isto pode ser constatado na Figura 2. 


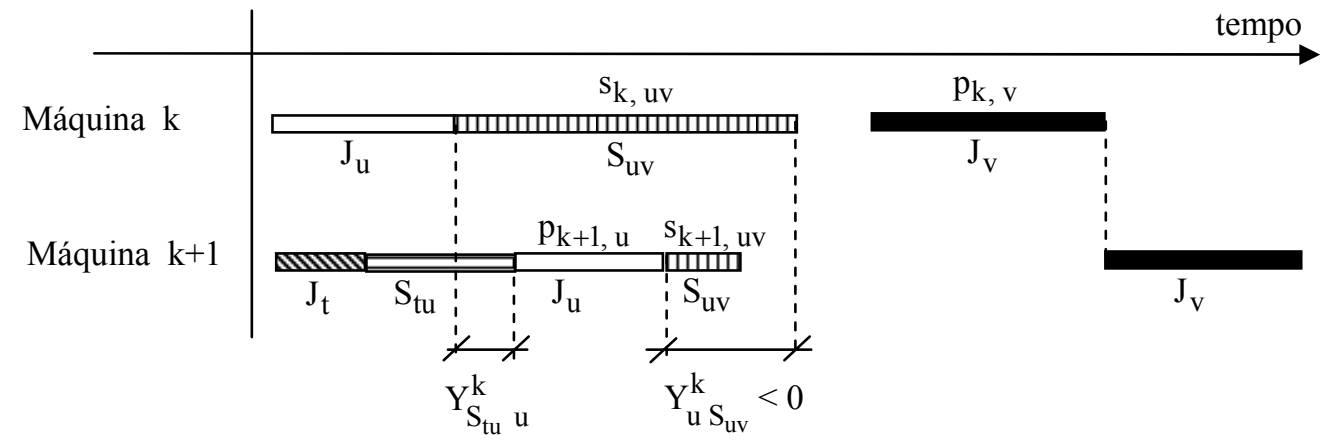

Figura 2 - Ilustração de uma situação em que $\mathrm{Y}_{\mathrm{u} \mathrm{S}}^{\mathrm{k}}<0$.

Substituindo $\mathrm{Y}_{\mathrm{u} \mathrm{S}_{\mathrm{uv}}^{\mathrm{k}}}^{\mathrm{de}}$ (6) na expressão (5) tem-se

$\mathrm{X}_{\mathrm{S}_{\mathrm{uv}} \mathrm{v}}^{\mathrm{k}}+\mathrm{p}_{\mathrm{k}, \mathrm{v}}+\mathrm{Y}_{\mathrm{S}_{\mathrm{uv}} \mathrm{v}}^{\mathrm{k}}=\mathrm{Y}_{\mathrm{S}_{\mathrm{tu}} \mathrm{u}}^{\mathrm{k}}+\mathrm{p}_{\mathrm{k}+1, \mathrm{u}}-\mathrm{s}_{\mathrm{k}, \mathrm{uv}}+\mathrm{s}_{\mathrm{k}+1, \mathrm{uv}}+\mathrm{X}_{\mathrm{S}_{\mathrm{uv}} \mathrm{v}}^{\mathrm{k}+1}$,

que leva a

$\mathrm{X}_{\mathrm{S}_{\mathrm{uv}} \mathrm{v}}^{\mathrm{k}}+\left(\mathrm{p}_{\mathrm{k}, \mathrm{v}}+\mathrm{s}_{\mathrm{k}, \mathrm{uv}}\right)+\mathrm{Y}_{\mathrm{S}_{\mathrm{uv}} \mathrm{v}}^{\mathrm{k}}=\mathrm{Y}_{\mathrm{S}_{\mathrm{tu}} \mathrm{u}}^{\mathrm{k}}+\left(\mathrm{p}_{\mathrm{k}+1, \mathrm{u}}+\mathrm{s}_{\mathrm{k}+1, \mathrm{uv}}\right)+\mathrm{X}_{\mathrm{S}_{\mathrm{uv}} \mathrm{v}}^{\mathrm{k}+1}$.

A expressão (7) é semelhante à tradicional expressão (1) que viabiliza, para duas tarefas sucessivas $J_{u}$ e $J_{v}$, as relações entre tempos de máquina parada e tempos de espera entre operações sucessivas de uma mesma tarefa, no problema em que os tempos de preparação das máquinas são incluídos nos tempos de processamento das tarefas. A diferença fundamental é que, na expressão (7) os tempos de máquina parada $X_{i j}^{q}$ referem-se à tarefa $\mathrm{J}_{\mathrm{V}}$ e à preparação $\mathrm{S}_{\mathrm{uv}}$ das máquinas para sua execução, enquanto que na expressão (1) tais tempos indicam o intervalo entre o término da tarefa $J_{u}$ e o início de $J_{v}$. Note-se também que os valores de $X_{i j}^{q}$ e $Y_{i j}^{q}$ mantêm a condição de serem sempre positivos ou nulos. Na expressão (7), $X_{i j}^{q}$ é a medida do intervalo de tempo entre o término de preparação da máquina e o início da execução da tarefa para a qual foi preparada, e $\mathrm{Y}_{\mathrm{ij}}^{\mathrm{q}}$ é o tempo de espera entre operações sucessivas de uma mesma tarefa, as quais apresentam entre si uma relação de precedência tecnológica direta.

Desta forma, pode-se completar a expressão (7) com as demais relações de viabilidade, conforme segue:

Se $\mathrm{X}_{\mathrm{S}_{\mathrm{uv}} \mathrm{v}}^{\mathrm{k}+1}>0$ então $\mathrm{Y}_{\mathrm{S}_{\mathrm{uv}} \mathrm{v}}^{\mathrm{k}}=0$, e

Se $\mathrm{Y}_{\mathrm{S}_{\mathrm{uv}} \mathrm{v}}^{\mathrm{k}}>0$ então $\quad \mathrm{X}_{\mathrm{S}_{\mathrm{uv}} \mathrm{k}}^{\mathrm{k}+1}=0$. 
A Figura 3 ilustra tais relações de viabilidade.

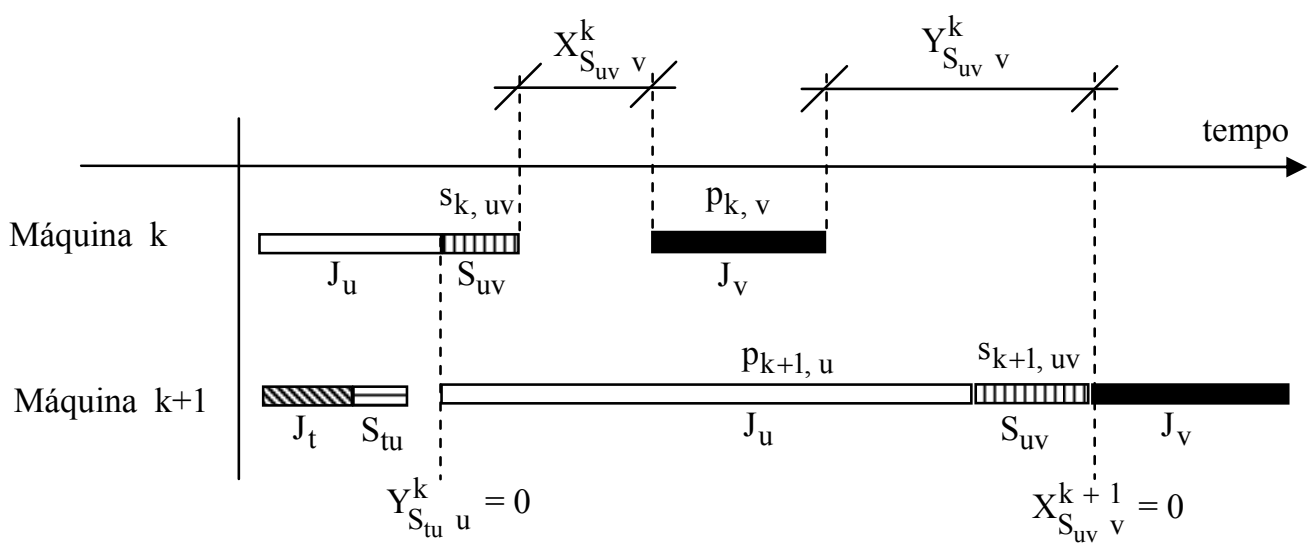

Figura 3 - Relações de viabilidade entre $S_{u v}$ e $J_{v}$.

Seja $\operatorname{UBX}_{\mathrm{S}_{\mathrm{uv}} \mathrm{v}}^{\mathrm{k}}$ um Limitante Superior de $\mathrm{X}_{\mathrm{S}_{\mathrm{uv}} \mathrm{v}}^{\mathrm{k}}$, ou seja, $\mathrm{X}_{\mathrm{S}_{\mathrm{uv}} \mathrm{v}}^{\mathrm{k}} \leq \mathrm{UBX}_{\mathrm{S}_{\mathrm{uv}} \mathrm{v}}^{\mathrm{k}}$, para $\mathrm{k}=1,2, \ldots ., \mathrm{m}$.

Sendo $\quad \mathrm{X}_{\mathrm{S}_{\mathrm{uv}} \mathrm{v}}^{\mathrm{k}} \leq \mathrm{UBX}_{\mathrm{S}_{\mathrm{uv}} \mathrm{v}}^{\mathrm{k}}$, adicionando-se $\left(\mathrm{p}_{\mathrm{k}, \mathrm{v}}+\mathrm{s}_{\mathrm{k}, \mathrm{uv}}\right)-\left(\mathrm{p}_{\mathrm{k}+1, \mathrm{u}}+\mathrm{s}_{\mathrm{k}+1, \mathrm{uv}}\right) \quad$ em ambos os termos da desigualdade, tem-se:

$\mathrm{X}_{\mathrm{S}_{\mathrm{uv}} \mathrm{v}}^{\mathrm{k}}+\left(\mathrm{p}_{\mathrm{k}, \mathrm{v}}+\mathrm{s}_{\mathrm{k}, \mathrm{uv}}\right)-\left(\mathrm{p}_{\mathrm{k}+1, \mathrm{u}}+\mathrm{s}_{\mathrm{k}+1, \mathrm{uv}}\right) \leq \mathrm{UBX}_{\mathrm{S}_{\mathrm{uv}} \mathrm{v}}^{\mathrm{k}}+\left(\mathrm{p}_{\mathrm{k}, \mathrm{v}}+\mathrm{s}_{\mathrm{k}, \mathrm{uv}}\right)-\left(\mathrm{p}_{\mathrm{k}+1, \mathrm{u}}+\mathrm{s}_{\mathrm{k}+1, \mathrm{uv}}\right)$.

A expressão de viabilidade (7) entre $\mathrm{S}_{\mathrm{uv}}$ e $\mathrm{J}_{\mathrm{v}}$ pode ser escrita como:

$\mathrm{X}_{\mathrm{S}_{\mathrm{uv}} \mathrm{v}}^{\mathrm{k}+1}-\mathrm{Y}_{\mathrm{S}_{\mathrm{uv}} \mathrm{v}}^{\mathrm{k}}=\mathrm{X}_{\mathrm{S}_{\mathrm{uv}} \mathrm{v}}^{\mathrm{k}}+\left(\mathrm{p}_{\mathrm{k}, \mathrm{v}}+\mathrm{s}_{\mathrm{k}, \mathrm{uv}}\right)-\left(\mathrm{p}_{\mathrm{k}+1, \mathrm{u}}+\mathrm{s}_{\mathrm{k}+1, \mathrm{uv}}\right)-\mathrm{Y}_{\mathrm{S}_{\mathrm{tu}} \mathrm{u}}^{\mathrm{k}}$.

Uma vez que, por definição, $\mathrm{Y}_{\mathrm{S}_{\mathrm{tu}} \mathrm{u}}^{\mathrm{k}} \geq 0$, tem-se:

$\mathrm{X}_{\mathrm{S}_{\mathrm{uv}} \mathrm{v}}^{\mathrm{k}+1}-\mathrm{Y}_{\mathrm{S}_{\mathrm{uv}} \mathrm{v}}^{\mathrm{k}} \leq \mathrm{X}_{\mathrm{S}_{\mathrm{uv}} \mathrm{v}}^{\mathrm{k}}+\left(\mathrm{p}_{\mathrm{k}, \mathrm{v}}+\mathrm{s}_{\mathrm{k}, \mathrm{uv}}\right)-\left(\mathrm{p}_{\mathrm{k}+1, \mathrm{u}}+\mathrm{s}_{\mathrm{k}+1, \mathrm{uv}}\right)$.

Utilizando-se as expressões (10) e (11) pode-se escrever que

$\mathrm{X}_{\mathrm{S}_{\mathrm{uv}} \mathrm{v}}^{\mathrm{k}+1}-\mathrm{Y}_{\mathrm{S}_{\mathrm{uv}} \mathrm{v}}^{\mathrm{k}} \leq \mathrm{UBX}_{\mathrm{S}_{\mathrm{uv}} \mathrm{v}}^{\mathrm{k}}+\left(\mathrm{p}_{\mathrm{k}, \mathrm{v}}+\mathrm{s}_{\mathrm{k}, \mathrm{uv}}\right)-\left(\mathrm{p}_{\mathrm{k}+1, \mathrm{u}}+\mathrm{s}_{\mathrm{k}+1, \mathrm{uv}}\right)$.

A expressão (12) fornece um limitante superior para a diferença entre as variáveis $X_{S_{u v}+1}^{k}$ e $\mathrm{Y}_{\mathrm{S}_{\mathrm{uv}} \mathrm{v}}^{\mathrm{k}}$, as quais não podem ser simultaneamente positivas, conforme as expressões (8) e (9). Desta forma, pode-se enunciar a seguinte proposição: 
Considere-se o problema de programação da produção flow shop permutacional com os tempos de preparação das máquinas separados dos tempos de processamento das tarefas, dependentes ou não da seqüência de execução das tarefas. Sejam $J_{u}$ e $J_{v}$ duas tarefas sucessivas quaisquer, e $\mathrm{S}_{\mathrm{uv}}$ a atividade de preparação das máquinas para execução de $\mathrm{J}_{\mathrm{v}}$. Seja $U_{B X} \mathrm{~S}_{\mathrm{uv}_{\mathrm{v}}}$ um limitante superior de $\mathrm{X}_{\mathrm{S}_{\mathrm{uv}} \mathrm{v}}^{\mathrm{k}}$, intervalo de tempo em $\mathrm{M}_{\mathrm{k}}$ entre o término da preparação $\mathrm{S}_{\mathrm{uv}}$ e o início de $\mathrm{J}_{\mathrm{v}}$. Analogamente, seja $\mathrm{UBX}_{\mathrm{S}_{\mathrm{uv}} \mathrm{v}}^{\mathrm{v}}$ um limitante superior de $\mathrm{X}_{\mathrm{S}_{\mathrm{uv}} \mathrm{v}}^{\mathrm{k}+1}$.

a) Se $\mathrm{UBX}_{\mathrm{S}_{\mathrm{uv}} \mathrm{v}}^{\mathrm{k}}+\left(\mathrm{p}_{\mathrm{k}, \mathrm{v}}+\mathrm{s}_{\mathrm{k}, \mathrm{uv}}\right)-\left(\mathrm{p}_{\mathrm{k}+1, \mathrm{u}}+\mathrm{s}_{\mathrm{k}+1, \mathrm{uv}}\right)>0$

$$
\begin{aligned}
& \text { então } \quad \mathrm{X}_{\mathrm{S}_{\mathrm{uv}} \mathrm{v}}^{\mathrm{k}+1} \leq \mathrm{UBX}_{\mathrm{S}_{\mathrm{uv}} \mathrm{v}}^{\mathrm{k}}+\left(\mathrm{p}_{\mathrm{k}, \mathrm{v}}+\mathrm{s}_{\mathrm{k}, \mathrm{uv}}\right)-\left(\mathrm{p}_{\mathrm{k}+1, \mathrm{u}}+\mathrm{s}_{\mathrm{k}+1, \mathrm{uv}}\right) e \\
& \mathrm{UBX}_{\mathrm{S}_{\mathrm{uv}} \mathrm{v}}^{\mathrm{k}+1}=\mathrm{UBX}_{\mathrm{S}_{\mathrm{uv}} \mathrm{v}}^{\mathrm{k}}+\left(\mathrm{p}_{\mathrm{k}, \mathrm{v}}+\mathrm{s}_{\mathrm{k}, \mathrm{uv}}\right)-\left(\mathrm{p}_{\mathrm{k}+1, \mathrm{u}}+\mathrm{s}_{\mathrm{k}+1, \mathrm{uv}}\right) .
\end{aligned}
$$

b) Caso contrário, se $\mathrm{UBX}_{\mathrm{S}_{\mathrm{uv}} \mathrm{v}}^{\mathrm{k}}+\left(\mathrm{p}_{\mathrm{k}, \mathrm{v}}+\mathrm{s}_{\mathrm{k}, \mathrm{uv}}\right)-\left(\mathrm{p}_{\mathrm{k}+1, \mathrm{u}}+\mathrm{s}_{\mathrm{k}+1, \mathrm{uv}}\right) \leq 0$ então $\quad \mathrm{X}_{\mathrm{S}_{\mathrm{uv}} \mathrm{v}}^{\mathrm{k}+1}=0$ e e $\mathrm{UBX}_{\mathrm{S}_{\mathrm{uv}} \mathrm{v}}^{\mathrm{k}+1}=0$.

\section{Prova da proposição:}

a) Por definição $X_{S_{u v}}^{k+1} \geq 0$.

- $\operatorname{Se} \mathrm{X}_{\mathrm{S}_{\mathrm{uv}} \mathrm{k}}^{\mathrm{k}+1}=0$ e $\mathrm{UBX}_{\mathrm{S}_{\mathrm{uv}}^{\mathrm{k}}}^{\mathrm{k}}+\left(\mathrm{p}_{\mathrm{k}, \mathrm{v}}+\mathrm{s}_{\mathrm{k}, \mathrm{uv}}\right)-\left(\mathrm{p}_{\mathrm{k}+1, \mathrm{u}}+\mathrm{s}_{\mathrm{k}+1, \mathrm{uv}}\right)>0$, obviamente $\mathrm{X}_{\mathrm{S}_{\mathrm{uv}}^{\mathrm{k}} \mathrm{v}}^{\mathrm{k}}<\mathrm{UBX}_{\mathrm{S}_{\mathrm{uv}} \mathrm{v}}^{\mathrm{k}}+\left(\mathrm{p}_{\mathrm{k}, \mathrm{v}}+\mathrm{s}_{\mathrm{k}, \mathrm{uv}}\right)-\left(\mathrm{p}_{\mathrm{k}+1, \mathrm{u}}+\mathrm{s}_{\mathrm{k}+1, \mathrm{uv}}\right)$.

- Se, por outro lado, $\mathrm{X}_{\mathrm{S}_{\mathrm{uv}} \mathrm{k}}^{\mathrm{v}+1}>0$, então pela expressão (8) $\mathrm{Y}_{\mathrm{S}_{\mathrm{uv}} \mathrm{v}}^{\mathrm{k}}=0$. Logo, utilizando-se a expressão (12) tem-se $\mathrm{X}_{\mathrm{S}_{\mathrm{uv}}^{\mathrm{k}} \mathrm{v}}^{\mathrm{1}} \leq \mathrm{UBX}_{\mathrm{S}_{\mathrm{uv}} \mathrm{v}}^{\mathrm{k}}+\left(\mathrm{p}_{\mathrm{k}, \mathrm{v}}+\mathrm{s}_{\mathrm{k}, \mathrm{uv}}\right)-\left(\mathrm{p}_{\mathrm{k}+1, \mathrm{u}}+\mathrm{s}_{\mathrm{k}+1, \mathrm{uv}}\right)$.

Portanto, $\mathrm{UBX}_{\mathrm{S}_{\mathrm{uv}}^{\mathrm{v}}}^{\mathrm{k}+1}=\mathrm{UBX}_{\mathrm{S}_{\mathrm{uv}} \mathrm{v}}^{\mathrm{k}}+\left(\mathrm{p}_{\mathrm{k}, \mathrm{v}}+\mathrm{s}_{\mathrm{k}, \mathrm{uv}}\right)-\left(\mathrm{p}_{\mathrm{k}+1, \mathrm{u}}+\mathrm{s}_{\mathrm{k}+1, \mathrm{uv}}\right)$.

b) A condição $\mathrm{UBX}_{\mathrm{S}_{\mathrm{uv}} \mathrm{v}}^{\mathrm{k}}+\left(\mathrm{p}_{\mathrm{k}, \mathrm{v}}+\mathrm{s}_{\mathrm{k}, \mathrm{uv}}\right)-\left(\mathrm{p}_{\mathrm{k}+1, \mathrm{u}}+\mathrm{s}_{\mathrm{k}+1, \mathrm{uv}}\right) \leq 0$ leva, a partir da expressão (12), a $\mathrm{X}_{\mathrm{S}_{\mathrm{uv}} \mathrm{k}}^{\mathrm{k}+1}-\mathrm{Y}_{\mathrm{S}_{\mathrm{uv}} \mathrm{v}}^{\mathrm{k}} \leq 0$.

Tendo em vista as relações de viabilidade (8) e (9), $\mathrm{X}_{\mathrm{S}_{\mathrm{uv}} \mathrm{v}}^{\mathrm{k}+1}$ e $\mathrm{Y}_{\mathrm{S}_{\mathrm{uv}} \mathrm{v}}^{\mathrm{k}}$ são valores positivos ou nulos, mas que não podem ser simultaneamente positivos. Desta forma, a desigualdade $\mathrm{X}_{\mathrm{S}_{\mathrm{uv}} \mathrm{v}}^{\mathrm{k}+1}-\mathrm{Y}_{\mathrm{S}_{\mathrm{uv}} \mathrm{v}}^{\mathrm{k}} \leq 0$ somente é válida se $\mathrm{X}_{\mathrm{S}_{\mathrm{uv}}^{\mathrm{v}} \mathrm{v}}^{\mathrm{k}}=0$.

Portanto, $\mathrm{UBX}_{\mathrm{S}_{\mathrm{uv}} \mathrm{v}}^{\mathrm{k}+1}=0$. 
Com fundamento na proposição acima, pode-se estabelecer a seguinte propriedade:

No problema FSP-TPS com m máquinas e $n$ tarefas, sendo $J_{u}$ e $J_{v}$ duas tarefas sucessivas quaisquer, $S_{u v}$ a atividade de preparação da máquina $M_{k+1}$ para execução de $J_{v}$, e $X_{S_{u v}}^{k+1}$ o intervalo de tempo em $M_{k+1}$ entre o término da preparação $S_{u v}$ e o início de $J_{v}$, então $U B X_{S_{u v} v}^{k+1}$ é um Limitante Superior de $X_{S_{u v}}^{k+1}$, dado por:

$\mathrm{UBX}_{\mathrm{S}_{\mathrm{uv}} \mathrm{v}}^{\mathrm{k}+1}=\operatorname{Max}\left[0, \mathrm{UBX}_{\mathrm{S}_{\mathrm{uv}} \mathrm{v}}^{\mathrm{k}}+\left(\mathbf{p}_{\mathrm{k}, \mathrm{v}}+\mathbf{s}_{\mathrm{k}, \mathrm{uv}}\right)-\left(\mathbf{p}_{\mathrm{k}+1, \mathrm{u}}+\mathbf{s}_{\mathrm{k}+1, \mathrm{uv}}\right)\right]$

$\mathrm{k}=1,2, \ldots . ., \mathrm{m}-1 \quad \operatorname{com} \quad \mathrm{UBX}_{\mathrm{S}_{\mathrm{uv}} \mathrm{v}}^{1}=\mathbf{0}$

A expressão (13) permite calcular o limitante superior $\mathrm{UBX}_{\mathrm{S}_{\mathrm{uv}} \mathrm{v}}^{\mathrm{v}}$ para uma máquina qualquer, de maneira recorrente, a partir de $\mathrm{UBX}_{\mathrm{S}_{\mathrm{uv}} \mathrm{v}}{ }=0$, uma vez que na primeira máquina os intervalos de tempo $\mathrm{X}_{\mathrm{S}_{\mathrm{uv}} \mathrm{v}}^{1}$ são nulos.

\section{Aplicação da Propriedade}

Uma aplicação da propriedade apresentada é descrita a seguir.

Considerando uma seqüência qualquer $\sigma$ das n tarefas, a Duração Total da Programação (makespan) pode ser calculada pela seguinte expressão:

$M(\sigma)=\sum_{j=1}^{n} p_{m[j]}+\sum_{j=0}^{n-1} s_{m[j+1]}+\sum_{j=0}^{n-1} X_{[j+1]}^{m}$

onde

$\mathrm{p}_{\mathrm{m}[\mathrm{j}]}=$ tempo de processamento, na última máquina, da tarefa que ocupa a j-ésima posição na seqüência $\sigma$;

$\mathrm{s}_{\mathrm{m}[\mathrm{j}+1]}=$ tempo de preparação da última máquina para execução da tarefa que ocupa a posição $(j+1)$ na seqüência $\sigma$;

$\mathrm{X}_{[\mathrm{j}+1]}^{\mathrm{m}}=$ intervalo de tempo, na última máquina, entre o término de sua preparação e o início da execução da tarefa que ocupa a posição $(j+1)$ na seqüência $\sigma$; sendo que

$\mathrm{j}=0$ corresponde a uma tarefa fictícia com tempos de processamento e preparação nulos, e que ocupa sempre a posição anterior à primeira tarefa em qualquer seqüência das $n$ tarefas.

A partir das expressões (13) e (14) pode-se calcular um Limitante Superior do makespan $\mathrm{M}(\sigma)$, conforme segue: 


$$
\operatorname{UBM}(\sigma)=\sum_{j=1}^{n} p_{m[j]}+\sum_{j=0}^{n-1} s_{m[j+1]}+\sum_{j=0}^{n-1} \operatorname{UBX}_{[j+1]}^{m}
$$

O primeiro termo da direita na expressão (15) é constante e independe da seqüência $\sigma$. Para o caso em que os tempos de preparação das máquinas são independentes, o segundo termo também será constante e quando os tempos de preparação das máquinas forem dependentes da seqüência das tarefas, tanto o segundo quanto o terceiro termo dependem da seqüência considerada.

Entretanto, para ambas as situações o problema original de programação da produção, quando se deseja minimizar o makespan, pode ser resolvido heuristicamente por meio da solução de um problema transformado, no qual objetiva-se determinar a seqüência das tarefas que minimiza o limitante superior $\operatorname{UBM}(\sigma)$.

Tal problema transformado é análogo ao problema cíclico assimétrico do caixeiro-viajante $(A T S P)$ ilustrado na Figura 4.

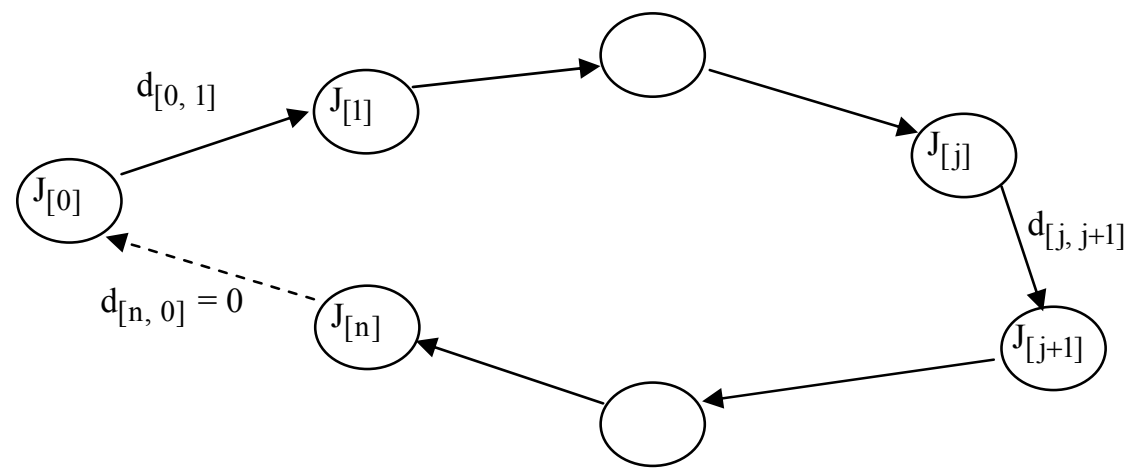

Figura 4 - Analogia do problema original de programação da produção com o problema cíclico assimétrico do caixeiro-viajante.

No problema ilustrado pela Figura 4, a cidade-base seria a tarefa fictícia $\mathrm{J}_{[0]}$, as cidades a serem visitadas seriam as $\mathrm{n}$ tarefas, e uma rota viável seria a seqüência $\mathrm{J}_{[0]}, \mathrm{J}_{[1]}, \ldots, \mathrm{J}_{[\mathrm{j}]}$, $\mathrm{J}_{[\mathrm{j}+1]}, \ldots, \mathrm{J}_{[\mathrm{n}]}, \mathrm{J}_{[0]}$, onde $\mathrm{J}_{[\mathrm{j}]}$ representa a tarefa que ocupa a j-ésima posição na seqüência $\sigma$.

No caso de tempos de preparação das máquinas dependentes da seqüência das tarefas, a distância entre duas cidades (tarefas) $d_{[j, j+1]}$ seria a soma $\left(s_{m[j+1]}+U B X_{[j+1]}^{m}\right)$, para $j=0$, $1,2, \ldots . .,(n-1)$. Se os tempos de preparação das máquinas forem independentes da seqüência das tarefas, tal distância seria somente o limitante superior $\mathrm{UBX}_{[\mathrm{j}+1]}^{\mathrm{m}}$.

A modelagem de um problema de programação da produção flow shop por meio de um problema do caixeiro-viajante tem ocorrido freqüentemente. 
Framinan, Gupta \& Leisten (2004) apresentam uma revisão e classificação de métodos heurísticos para o problema clássico de programação da produção flow shop permutacional (com os tempos de preparação das máquinas incluídos nos tempos de processamento das tarefas) tendo como medida de desempenho a minimização do makespan. Nesse artigo, são citados quatro trabalhos que buscam resolver heuristicamente tal problema a partir da solução de um problema de caixeiro-viajante: Gupta (1968), Stinson \& Smith (1982), Widmer \& Hertz (1989), e Moccellin (1995). Uma diferença básica entre esses trabalhos consiste na forma pela qual são calculadas as distâncias entre as operações sucessivas das tarefas na última máquina do flow shop. A analogia proposta neste trabalho entre o problema de programação da produção FSP-TPS e do caixeiro-viajante (ATSP) é similar àquela apresentada em Moccellin (1995).

Conforme já mencionado, os métodos SETUP e TOTAL (Simons Jr., 1992), e HYBRID (Rios-Mercado \& Bard, 1999b) fundamentam-se no problema assimétrico do caixeiroviajante para obter soluções heurísticas do problema FSP-TPS com tempos de preparação das máquinas dependentes da seqüência de execução das tarefas.

Os trabalhos acima citados, que utilizam analogia com o problema do caixeiro-viajante fazem parte de uma classe de problemas flow shop com buffer não-limitado, uma vez que as tarefas não apresentam restrições de armazenagem entre as suas operações sucessivas nas diversas máquinas. No caso de buffer limitado existe um número máximo de tarefas que podem ser armazenadas aguardando os respectivos processamentos nas máquinas. Outras variações do ambiente flow shop com restrições quanto ao processamento das tarefas denominam-se blocking e no-wait flow shop. No primeiro caso, não existe a possibilidade de armazenar as tarefas entre as suas operações sucessivas nas máquinas, de forma que uma tarefa que já tenha sido processada em uma determinada máquina não pode liberá-la, portanto bloqueando-a, até que a máquina da próxima operação esteja disponível. No segundo caso, uma tarefa uma vez iniciada não pode ser interrompida até o seu completo processamento, ou seja, não deve existir tempo de espera entre as suas operações sucessivas. Em uma recente revisão da literatura, Bagchi, Gupta \& Sriskandarajah (2006) tratam desses ambientes de produção específicos, além de outros como o flow shop flexível e o automatizado, apresentando diversos trabalhos e formulações desses problemas de produção flow shop baseados em analogias com o problema do caixeiro-viajante.

\section{Análise Estatística da analogia entre os problemas FSP-TPS e ATSP}

A partir dos fundamentos apresentados em Mood, Graybill \& Boes (1974), pode-se efetuar a análise estatística apresentada a seguir.

A analogia entre os problemas FSP-TPS e ATSP pode ser considerada adequada se os espaços de soluções dos mesmos forem equivalentes, dentro de uma faixa aceitável de aproximação. Isto significa que, se os espaços forem totalmente equivalentes, as soluções ótimas dos dois problemas também serão, ou seja, a seqüência de tarefas que minimiza o makespan (função-objetivo do problema original de programação da produção) é a mesma seqüência que minimiza o limitante superior $\operatorname{UBM}(\sigma)$ (função-objetivo do problema cíclico assimétrico do caixeiro-viajante).

A condição de total equivalência pode ser estabelecida conforme segue: 
Seja $\operatorname{DR}(\sigma)$ o Desvio Relativo entre o limitante superior $\operatorname{UBM}(\sigma)$ e o makespan $\mathrm{M}(\sigma)$ de uma seqüência genérica $\sigma$, dado por:

$$
\operatorname{DR}(\sigma)=\frac{\operatorname{UBM}(\sigma)-\mathrm{M}(\sigma)}{\mathrm{M}(\sigma)} \quad \text { que leva a } \quad \operatorname{UBM}(\sigma)=(1+\operatorname{DR}(\sigma)) \mathrm{M}(\sigma)
$$

Assim, a total equivalência é condicionada a $\operatorname{DR}(\sigma)$ assumir o mesmo valor (constante) para todas as seqüências possíveis das n tarefas, ou seja, (n!) seqüências.

Obviamente, tal condição de total equivalência não é satisfeita, uma vez que os Desvios Relativos podem assumir valores diferentes. Por outro lado, uma equivalência aproximada pode ser obtida substituindo o valor $\operatorname{DR}(\sigma)$ por uma estimativa que incorpora a sua variabilidade, por meio da expressão que segue:

Estimativa $[\mathrm{DR}(\sigma)]=\overline{\mathrm{DR}} \pm \mathrm{S}_{\mathrm{DR}}$, onde

$\overline{\mathrm{DR}}=$ Desvio Relativo Médio considerando o espaço de soluções constituído das (n!) seqüências, calculado por $\overline{\mathrm{DR}}=\frac{1}{\mathrm{n} !} \sum_{\mathrm{i}=1}^{\mathrm{n} !} \mathrm{DR}(\sigma)_{\mathrm{i}}$;

$S_{\mathrm{DR}}=$ Desvio Padrão do desvio relativo $\mathrm{DR}(\sigma)$, dado por $\mathrm{S}_{\mathrm{DR}}=\sqrt{\frac{\sum_{\mathrm{i}=1}^{\mathrm{n} !}\left(\mathrm{DR}(\sigma)_{\mathrm{i}}-\overline{\mathrm{DR}}\right)^{2}}{n !-1}}$.

Utilizando a expressão (17) em (16) obtém-se uma relação aproximada entre o limitante superior $\operatorname{UBM}(\sigma)$ e o makespan $\mathrm{M}(\sigma)$, ou seja:

Estimativa $[\mathrm{UBM}(\sigma)]=\left(1+\overline{\mathrm{DR}} \pm \mathrm{S}_{\mathrm{DR}}\right) \mathrm{M}(\sigma)$

A expressão (18) pode ser entendida como uma estatística que reflete o "grau de equivalência" entre os espaços de soluções dos dois problemas (programação da produção e caixeiro-viajante). É importante notar que esse grau de equivalência depende somente da amplitude do desvio padrão $\mathrm{S}_{\mathrm{DR}}$. Quanto menor o desvio padrão, maior o grau de equivalência. $\mathrm{S}_{\mathrm{DR}}=0$ leva a $\overline{\mathrm{DR}}=\mathrm{DR}(\sigma)_{\mathrm{i}}$ para todo $\mathrm{i}=1,2, \ldots, \mathrm{n}$ !, ou seja, um Desvio Relativo $\operatorname{DR}(\sigma)$ constante, que seria a condição de total equivalência entre os espaços de soluções.

\subsection{Um Limitante Superior do Desvio Padrão $S_{D R}$}

Com o objetivo de avaliar a influência dos parâmetros do problema de programação da produção (número de máquinas, número de tarefas, tempos de processamento das tarefas e tempos de preparação das máquinas), foi deduzida uma expressão que fornece um limitante superior do Desvio Padrão $\mathrm{S}_{\mathrm{DR}}$ em função dos mencionados parâmetros.

Para tanto, considere-se o problema FSP-TPS com m máquinas e $\mathrm{n}$ tarefas, e duas variáveis aleatórias independentes $\mathrm{P}$ e $\mathrm{S}$, sendo 
$\mathrm{P}=$ Variável aleatória dos tempos de processamento das tarefas, com média $\mu[\mathrm{P}]$ e variância $\operatorname{var}[\mathrm{P}]$;

$\mathrm{S}=$ Variável aleatória dos tempos de preparação das máquinas, com média $\mu[\mathrm{S}]$ e variância $\operatorname{var}[\mathrm{S}]$.

Sejam MIN[P] e MAX[P] o valor mínimo e máximo, respectivamente, dos tempos de processamento das tarefas. Analogamente, $\operatorname{MIN}[\mathrm{S}]$ e $\mathrm{MAX}[\mathrm{S}]$ para os tempos de preparação das máquinas.

Desta forma, o makespan $\mathrm{M}(\sigma)$ e seu limitante superior $\operatorname{UBM}(\sigma)$, os intervalos de tempo $\mathrm{X}_{\mathrm{S}_{\mathrm{uv}} \mathrm{v}}^{\mathrm{k}+1}$ e os limitantes superiores $\mathrm{UBX}_{\mathrm{S}_{\mathrm{uv}} \mathrm{v}}^{\mathrm{k}+1}$, assim como o Desvio Relativo $\mathrm{DR}(\sigma)$, serão variáveis aleatórias dependentes de $\mathrm{P}$ e $\mathrm{S}$.

\subsubsection{Variável $\mathrm{UBX}_{\mathrm{S}_{\mathrm{uv}} \mathrm{v}}^{\mathrm{k}+1}$}

Conforme a expressão (13),

$$
\mathrm{UBX}_{\mathrm{S}_{\mathrm{uv}} \mathrm{v}}^{\mathrm{k}+1}=\operatorname{Max}\left[0, \mathrm{UBX}_{\mathrm{S}_{\mathrm{uv}} \mathrm{v}}^{\mathrm{k}}+\left(\mathrm{p}_{\mathrm{k}, \mathrm{v}}+\mathrm{s}_{\mathrm{k}, \mathrm{uv}}\right)-\left(\mathrm{p}_{\mathrm{k}+1, \mathrm{u}}+\mathrm{s}_{\mathrm{k}+1, \mathrm{uv}}\right)\right]
$$

$\mathrm{k}=1,2, \ldots ., \mathrm{m}-1 \quad$ com $\quad \mathrm{UBX}_{\mathrm{S}_{\mathrm{uv}} \mathrm{v}}^{1}=0$.

Portanto, para k=1 tem-se: $\mathrm{UBX}_{\mathrm{S}_{\mathrm{uv}} \mathrm{v}}^{2}=\operatorname{Max}\left[0,\left(\mathrm{p}_{1, \mathrm{v}}+\mathrm{s}_{1, \mathrm{uv}}\right)-\left(\mathrm{p}_{2, \mathrm{u}}+\mathrm{s}_{2, \mathrm{uv}}\right)\right]$

onde $\Delta_{1}(\mathrm{P}+\mathrm{S})=\left(\mathrm{p}_{1, \mathrm{v}}+\mathrm{s}_{1, \mathrm{uv}}\right)-\left(\mathrm{p}_{2, \mathrm{u}}+\mathrm{s}_{2, \text { uv }}\right)$ é uma variável aleatória (v.a.) com

média $\mu\left[\Delta_{1}(\mathrm{P}+\mathrm{S})\right]=(\mu[\mathrm{P}]+\mu[\mathrm{S}])-(\mu[\mathrm{P}]+\mu[\mathrm{S}])=0$, e

variância $\operatorname{var}\left[\Delta_{1}(\mathrm{P}+\mathrm{S})\right]=(\operatorname{var}[\mathrm{P}]+\operatorname{var}[\mathrm{S}])+(\operatorname{var}[\mathrm{P}]+\operatorname{var}[\mathrm{S}])=2(\operatorname{var}[\mathrm{P}]+\operatorname{var}[\mathrm{S}])$.

A v.a. $\Delta_{1}(\mathrm{P}+\mathrm{S})$ distribui-se simetricamente em relação ao valor zero, assumindo valores no intervalo $\operatorname{MIN}\left[\Delta_{1}(\mathrm{P}+\mathrm{S})\right]$ a $\operatorname{MAX}\left[\Delta_{1}(\mathrm{P}+\mathrm{S})\right]$, onde

$\operatorname{MIN}\left[\Delta_{1}(\mathrm{P}+\mathrm{S})\right]=(\operatorname{MIN}[\mathrm{P}]+\operatorname{MIN}[\mathrm{S}])-(\operatorname{MAX}[\mathrm{P}]+\operatorname{MAX}[\mathrm{S}])=\operatorname{MIN}[\mathrm{P}+\mathrm{S}]-\operatorname{MAX}[\mathrm{P}+\mathrm{S}]$, e $\operatorname{MAX}\left[\Delta_{1}(\mathrm{P}+\mathrm{S})\right]=\operatorname{MAX}[\mathrm{P}+\mathrm{S}]-\mathrm{MIN}[\mathrm{P}+\mathrm{S}]$

Assim, $\mathrm{UBX}_{\mathrm{S}_{\mathrm{uv}} \mathrm{v}}^{2}=\operatorname{Max}\left[0, \Delta_{1}(\mathrm{P}+\mathrm{S})\right]$ será uma v.a. com as seguintes características:

- Assume valores no intervalo $\left[0, \operatorname{MAX}\left[\Delta_{1}(\mathrm{P}+\mathrm{S})\right]\right]$;

- $0<\mu\left[\mathrm{UBX}_{\mathrm{S}_{\mathrm{uv}} \mathrm{v}}^{2}\right]<\operatorname{MAX}\left[\Delta_{1}(\mathrm{P}+\mathrm{S})\right]$, portanto $\operatorname{MAX}[\mathrm{P}+\mathrm{S}]-\mathrm{MIN}[\mathrm{P}+\mathrm{S}]$ é um limitante superior da média de $\mathrm{UBX}_{\mathrm{S}_{\mathrm{uv}} \mathrm{v}}^{2}$; 
- $\operatorname{var}\left[\mathrm{UBX}_{\mathrm{S}_{\mathrm{uv}} \mathrm{v}}^{2}\right]<\operatorname{var}\left[\Delta_{1}(\mathrm{P}+\mathrm{S})\right]$, portanto $2(\operatorname{var}[\mathrm{P}]+\operatorname{var}[\mathrm{S}])$ é um limitante superior da variância de $\mathrm{UBX}_{\mathrm{S}_{\mathrm{uv}} \mathrm{v}}^{2}$.

Para k=2, $\quad \mathrm{UBX}_{\mathrm{S}_{\mathrm{uv}} \mathrm{v}}^{3}=\operatorname{Max}\left[0, \mathrm{UBX}_{\mathrm{S}_{\mathrm{uv}} \mathrm{v}}^{2}+\left(\mathrm{p}_{2, \mathrm{v}}+\mathrm{s}_{2, \mathrm{uv}}\right)-\left(\mathrm{p}_{3, \mathrm{u}}+\mathrm{s}_{3, \mathrm{uv}}\right)\right]$

onde $\Delta_{2}(\mathrm{P}+\mathrm{S})=\left(\mathrm{p}_{2, \mathrm{v}}+\mathrm{s}_{2, \text { uv }}\right)-\left(\mathrm{p}_{3, \mathrm{u}}+\mathrm{s}_{3, \text { uv }}\right)$ é uma v.a. com a mesma distribuição de probabilidades de $\Delta_{1}(\mathrm{P}+\mathrm{S})$. Convém observar que $\Delta_{1}(\mathrm{P}+\mathrm{S})$ e $\Delta_{2}(\mathrm{P}+\mathrm{S})$ são variáveis aleatórias independentes, assim como $\mathrm{UBX}_{\mathrm{S}_{\mathrm{uv}} \mathrm{v}}^{2}$ e $\Delta_{2}(\mathrm{P}+\mathrm{S})$.

Analogamente a $\mathrm{UBX}_{\mathrm{S}_{\mathrm{uv}} \mathrm{v}}^{2}$, a v.a. $\mathrm{UBX}_{\mathrm{S}_{\mathrm{uv}} \mathrm{v}}^{3}=\operatorname{Max}\left[0, \mathrm{UBX}_{\mathrm{S}_{\mathrm{uv}} \mathrm{v}}^{2}+\Delta_{2}(\mathrm{P}+\mathrm{S})\right]$ apresenta as características que seguem:

- Assume valores no intervalo $\left[0, \operatorname{MAX}\left[\mathrm{UBX}_{\mathrm{S}_{\mathrm{uv}} \mathrm{v}}^{3}\right]\right]$, onde

$$
\begin{aligned}
& \operatorname{MAX}\left[\operatorname{UBX}_{\mathrm{S}_{\mathrm{uv}} \mathrm{v}}^{3}\right]=\operatorname{MAX}\left[\mathrm{UBX}_{\mathrm{S}_{\mathrm{uv}} \mathrm{v}}^{2}\right]+\operatorname{MAX}\left[\Delta_{2}(\mathrm{P}+\mathrm{S})\right], \text { ou seja, } \\
& \operatorname{MAX}\left[\mathrm{UBX}_{\mathrm{S}_{\mathrm{uv}} \mathrm{v}}^{3}\right]=2(\operatorname{MAX}[\mathrm{P}+\mathrm{S}]-\mathrm{MIN}[\mathrm{P}+\mathrm{S}])
\end{aligned}
$$

- $0<\mu\left[\mathrm{UBX}_{\mathrm{S}_{\mathrm{uv}} \mathrm{v}}^{3}\right]<\operatorname{MAX}\left[\mathrm{UBX}_{\mathrm{S}_{\mathrm{uv}} \mathrm{v}}^{3}\right]$, portanto $2(\mathrm{MAX}[\mathrm{P}+\mathrm{S}]-\mathrm{MIN}[\mathrm{P}+\mathrm{S}])$ é um limitante superior da média de $\mathrm{UBX}_{\mathrm{S}_{\mathrm{uv}} \mathrm{v}}^{3}$;

- $\operatorname{var}\left[\mathrm{UBX}_{\mathrm{S}_{\mathrm{uv}} \mathrm{v}}^{3}\right]<\operatorname{var}\left[\mathrm{UBX}_{\mathrm{S}_{\mathrm{uv}} \mathrm{v}}^{2}\right]+\operatorname{var}\left[\Delta_{2}(\mathrm{P}+\mathrm{S})\right]<\operatorname{var}\left[\Delta_{1}(\mathrm{P}+\mathrm{S})\right]+\operatorname{var}\left[\Delta_{2}(\mathrm{P}+\mathrm{S})\right]$, portanto $4(\operatorname{var}[\mathrm{P}]+\operatorname{var}[\mathrm{S}])$ é um limitante superior da variância de $\mathrm{UBX}_{\mathrm{S}_{\mathrm{uv}} \mathrm{v}}^{3}$.

Seguindo um procedimento análogo, pode-se estabelecer os seguintes limitantes superiores para a média e variância de $\mathrm{UBX}_{\mathrm{S}_{\mathrm{uv}} \mathrm{v}}^{\mathrm{k}+1}(\mathrm{k}=1,2, \ldots . ., \mathrm{m}-1)$ :

$0<\mu\left[\mathrm{UBX}_{\mathrm{S}_{\mathrm{uv}}^{\mathrm{v}} \mathrm{v}}^{\mathrm{k}}\right]<\mathrm{k}(\mathrm{MAX}[\mathrm{P}+\mathrm{S}]-\mathrm{MIN}[\mathrm{P}+\mathrm{S}]) ;$

$\operatorname{var}\left[\mathrm{UBX}_{\mathrm{S}_{\mathrm{uv}}^{\mathrm{v}} \mathrm{k}+1}^{\mathrm{k}}\right]<2 \mathrm{k}(\operatorname{var}[\mathrm{P}]+\operatorname{var}[\mathrm{S}])$,

onde $J_{u}$ e $J_{v}$ são duas tarefas sucessivas quaisquer. 


\subsubsection{Variável $\operatorname{UBM}(\sigma)$}

Conforme a expressão (15),

$\operatorname{UBM}(\sigma)=\sum_{j=1}^{n} p_{m[j]}+\sum_{j=0}^{n-1} s_{m[j+1]}+\sum_{j=0}^{n-1} U_{B X}^{m}[j+1]$, lembrando que

$\mathrm{p}_{\mathrm{m}[\mathrm{j}]}=$ tempo de processamento, na última máquina, da tarefa que ocupa a j-ésima posição na seqüência $\sigma$;

$\mathrm{s}_{\mathrm{m}[\mathrm{j}+1]}=$ tempo de preparação da última máquina para execução da tarefa que ocupa a posição $(\mathrm{j}+1)$ na seqüência $\sigma$;

$\mathrm{X}_{[\mathrm{j}+1]}^{\mathrm{m}}=$ intervalo de tempo, na última máquina, entre o término de sua preparação e o início da execução da tarefa que ocupa a posição $(j+1)$ na seqüência $\sigma$; sendo que

$\mathrm{j}=0 \quad$ corresponde a uma tarefa fictícia com tempos de processamento e preparação nulos, e que ocupa sempre a posição anterior à primeira tarefa em qualquer seqüência das $n$ tarefas.

Tendo-se que $\mathrm{p}_{\mathrm{m}[0]}=0$, a expressão (15) pode ser escrita como:

$$
\begin{aligned}
& \operatorname{UBM}(\sigma)=p_{m[0]}+\sum_{j=1}^{n} p_{m[j]}+\sum_{j=0}^{n-1} s_{m[j+1]}+\sum_{j=0}^{n-1} \operatorname{UBX}_{[j+1]}^{m}, \text { ou seja, } \\
& \operatorname{UBM}(\sigma)=\sum_{j=0}^{n-1} p_{m[j]}+p_{m[n]}+\sum_{j=0}^{n-1} s_{m[j+1]}+\sum_{j=0}^{n-1} U_{B X}^{m}[j+1] \text {, que leva a } \\
& \operatorname{UBM}(\sigma)=\sum_{j=0}^{n-1}\left(p_{m[j]}+s_{m[j+1]}\right)+p_{m[n]}+\sum_{j=0}^{n-1} \operatorname{UBX}_{[j+1]}^{m} .
\end{aligned}
$$

Da teoria estatística, considerando $Z_{1}, Z_{2}, \ldots, Z_{N}$ um conjunto de $N$ variáveis aleatórias, tem-se:

a média $\mu\left[\sum_{i=1}^{N} Z_{i}\right]=\sum_{i=1}^{N} \mu\left[Z_{i}\right]$

e a variância $\operatorname{var}\left[\sum_{i=1}^{N} Z_{i}\right]=\sum_{i=1}^{N} \operatorname{var}\left[Z_{i}\right]+2 \sum_{i<j} \sum_{j} \operatorname{cov}\left[Z_{i}, Z_{j}\right]$.

\section{Média de $\operatorname{UBM}(\sigma)$}

A partir das expressões (21) e (22),

$$
\mu[\operatorname{UBM}(\sigma)]=\sum_{j=0}^{n-1} \mu\left[\mathrm{p}_{m[j]}+\mathrm{s}_{m[j+1]}\right]+\mu\left[\mathrm{p}_{m[n]}\right]+\sum_{j=0}^{\mathrm{n}-1} \mu\left[\mathrm{UBX}_{[\mathrm{j}+1]}^{\mathrm{m}}\right] .
$$


Uma vez que $\mu\left[\mathrm{p}_{\mathrm{m}[0]}\right]=0, \mu\left[\mathrm{s}_{\mathrm{m}[1]}\right]=\mu[\mathrm{S}], \quad \mu\left[\mathrm{p}_{\mathrm{m}[\mathrm{n}]}\right]=\mu[\mathrm{P}]$ e $\mu\left[\mathrm{p}_{\mathrm{m}[\mathrm{j}]}+\mathrm{s}_{\mathrm{m}[\mathrm{j}+1]}\right]=\mu\left[\mathrm{p}_{\mathrm{m}[\mathrm{j}]}\right]+\mu\left[\mathrm{s}_{\mathrm{m}[\mathrm{j}+1]}\right]=\mu[\mathrm{P}]+\mu[\mathrm{S}] \quad \forall \mathrm{j}=1, \ldots, \mathrm{n}-1$, pode-se escrever $\mu[\operatorname{UBM}(\sigma)]=\mathrm{n}(\mu[\mathrm{P}]+\mu[\mathrm{S}])+\sum_{\mathrm{j}=0}^{\mathrm{n}-1} \mu\left[\mathrm{UBX}_{[\mathrm{j}+1]}^{\mathrm{m}}\right]$.

A expressão (19) fornece um limitante superior da média $\mu\left[\mathrm{UBX}_{\mathrm{S}_{\mathrm{uv}} \mathrm{v}}^{\mathrm{k}+1}\right]$ para todo $\mathrm{k}=1,2, \ldots . ., \mathrm{m}-1$, e qualquer par $\left(\mathrm{J}_{\mathrm{u}}, \mathrm{J}_{\mathrm{v}}\right)$ de tarefas sucessivas. Portanto, para a última máquina $\mathrm{m}$,

$0<\mu\left[\operatorname{UBX}_{[\mathrm{j}+1]}^{\mathrm{m}}\right]<(\mathrm{m}-1)(\operatorname{MAX}[\mathrm{P}+\mathrm{S}]-\mathrm{MIN}[\mathrm{P}+\mathrm{S}]) \quad \forall \mathrm{j}=0,1, \ldots, \mathrm{n}-1$.

A partir das expressões (24) e (25) pode-se estabelecer os seguintes limitantes para a média $\mu[\operatorname{UBM}(\sigma)]$ :

Limitante superior

$\operatorname{LS}(\mu[\operatorname{UBM}(\sigma)])=\mathrm{n}(\mu[\mathrm{P}]+\mu[\mathrm{S}]+(\mathrm{m}-1)(\operatorname{MAX}[\mathrm{P}+\mathrm{S}]-\mathrm{MIN}[\mathrm{P}+\mathrm{S}]))$.

Limitante inferior

LI $(\mu[\operatorname{UBM}(\sigma)])=\mathrm{n}(\mu[\mathrm{P}]+\mu[\mathrm{S}])$.

\section{Variância de $\operatorname{UBM}(\sigma)$}

Antes de se apresentar uma expressão para a $\operatorname{var}[\operatorname{UBM}(\sigma)]$, deve-se verificar as eventuais interdependências entre as variáveis aleatórias da expressão (21).

As variáveis aleatórias $\mathrm{P}$ (tempo de processamento das tarefas) e $\mathrm{S}$ (tempo de preparação das máquinas) são independentes, portanto as variáveis $\left(\mathrm{p}_{\mathrm{m}[\mathrm{j}]}+\mathrm{s}_{\mathrm{m}[\mathrm{j}+1]}\right)$ para $\mathrm{j}=0,1, \ldots, \mathrm{n}-1, \mathrm{e}$ $\mathrm{p}_{\mathrm{m}[\mathrm{n}]}$ são independentes entre si.

A expressão (13),

$$
\mathrm{UBX}_{\mathrm{S}_{\mathrm{uv}} \mathrm{v}}^{\mathrm{k}+1}=\operatorname{Max}\left[0, \mathrm{UBX}_{\mathrm{S}_{\mathrm{uv}} \mathrm{v}}^{\mathrm{k}}+\left(\mathrm{p}_{\mathrm{k}, \mathrm{v}}+\mathrm{s}_{\mathrm{k}, \mathrm{uv}}\right)-\left(\mathrm{p}_{\mathrm{k}+1, \mathrm{u}}+\mathrm{s}_{\mathrm{k}+1, \mathrm{uv}}\right)\right]
$$

pode ser escrita, de maneira equivalente, como

$$
\begin{aligned}
& \operatorname{UBX}_{[j+1]}^{\mathrm{k}+1}=\operatorname{Max}\left[0, \mathrm{UBX}_{[\mathrm{j}+1]}^{\mathrm{k}}+\left(\mathrm{p}_{\mathrm{k},[\mathrm{j}+1]}+\mathrm{s}_{\mathrm{k},[\mathrm{j}+1]}\right)-\left(\mathrm{p}_{\mathrm{k}+1,[j]}+\mathrm{s}_{\mathrm{k}+1,[\mathrm{j}+1]}\right)\right] \\
& \mathrm{k}=1,2, \ldots ., \mathrm{m}-1 \quad \text { com } \quad \operatorname{UBX}_{[\mathrm{j}+1]}^{1}=0, \operatorname{para} \mathrm{j}=0,1, \ldots, \mathrm{n}-1 .
\end{aligned}
$$

Assim, na última máquina $\mathrm{m}$, tem-se

$$
\mathrm{UBX}_{[\mathrm{j}+1]}^{\mathrm{m}}=\operatorname{Max}\left[0, \mathrm{UBX}_{[\mathrm{j}+1]}^{\mathrm{m}-1}+\left(\mathrm{p}_{\mathrm{m}-1,[\mathrm{j}+1]}+\mathrm{s}_{\mathrm{m}-1,[\mathrm{j}+1]}\right)-\left(\mathrm{p}_{\mathrm{m},[\mathrm{j}]}+\mathrm{s}_{\mathrm{m},[\mathrm{j}+1]}\right)\right] \text {. }
$$


Portanto, pode-se notar que os $\mathrm{n}$ pares de variáveis $\left(\left(\mathrm{p}_{\mathrm{m},[\mathrm{j}]}+\mathrm{s}_{\mathrm{m},[\mathrm{j}+1]}\right), \mathrm{UBX}_{[\mathrm{j}+1]}^{\mathrm{m}}\right)$ para $\mathrm{j}=0,1, \ldots, \mathrm{n}-1$, são dependentes, porém com correlação negativa. Desta forma, a covariância dessas variáveis é negativa. Os demais pares, ou seja, $\left(\left(\mathrm{p}_{\mathrm{m},[\mathrm{i}]}+\mathrm{s}_{\mathrm{m},[i+1]}\right), \mathrm{UBX}_{[\mathrm{j}+1]}^{\mathrm{m}}\right)$ para $i=0,1, \ldots, n-1, e j=0,1, \ldots, n-1$, com $i \neq j$, são variáveis aleatórias independentes.

Para concluir a verificação de interdependência entre as variáveis que fornecem o valor de $\operatorname{UBM}(\sigma)$, resta verificar as eventuais dependências no conjunto de $n$ variáveis $\operatorname{UBX}_{[j+1]}^{m}$, $\mathrm{j}=0,1, \ldots, \mathrm{n}-1$. Para tanto, considere-se que, em uma seqüência genérica $\sigma$ as tarefas $\mathrm{J}_{\mathrm{r}}$, $J_{t}, J_{u}$ e $J_{v}$ são sucessivas.

A expressão (13) para o par de tarefas sucessivas $\left(J_{r}, J_{t}\right)$ fica:

$$
\mathrm{UBX}_{\mathrm{S}_{\mathrm{rt}} \mathrm{t}}^{\mathrm{k}+1}=\operatorname{Max}\left[0, \mathrm{UBX}_{\mathrm{S}_{\mathrm{rt}} \mathrm{t}}^{\mathrm{k}}+\left(\mathrm{p}_{\mathrm{k}, \mathrm{t}}+\mathrm{s}_{\mathrm{k}, \mathrm{rt}}\right)-\left(\mathrm{p}_{\mathrm{k}+1, \mathrm{r}}+\mathrm{s}_{\mathrm{k}+1, \mathrm{rt}}\right)\right] \text {. }
$$

Para $\mathrm{k}=1$,

$$
\begin{aligned}
& \mathrm{UBX}_{\mathrm{S}_{\mathrm{rt}} \mathrm{t}}^{2}=\operatorname{Max}\left[0, \mathrm{UBX}_{\mathrm{S}_{\mathrm{rt}} \mathrm{t}}^{1}+\left(\mathrm{p}_{1, \mathrm{t}}+\mathrm{s}_{1, \mathrm{rt}}\right)-\left(\mathrm{p}_{2, \mathrm{r}}+\mathrm{s}_{2, \mathrm{rt}}\right)\right] \operatorname{com} \mathrm{UBX}_{\mathrm{S}_{\mathrm{rt}} \mathrm{t}}^{1}=0 \text {, portanto } \\
& \mathrm{UBX}_{\mathrm{S}_{\mathrm{rt}} \mathrm{t}}^{2}=\operatorname{Max}\left[0,\left(\mathrm{p}_{1, \mathrm{t}}+\mathrm{s}_{1, \mathrm{rt}}\right)-\left(\mathrm{p}_{2, \mathrm{r}}+\mathrm{s}_{2, \mathrm{rt}}\right)\right] .
\end{aligned}
$$

Para $\mathrm{k}=2$,

$$
\begin{aligned}
& \mathrm{UBX}_{\mathrm{S}_{\mathrm{rt}} \mathrm{t}}^{3}=\operatorname{Max}\left[0, \mathrm{UBX}_{\mathrm{S}_{\mathrm{rt}} \mathrm{t}}^{2}+\left(\mathrm{p}_{2, \mathrm{t}}+\mathrm{s}_{2, \mathrm{rt}}\right)-\left(\mathrm{p}_{3, \mathrm{r}}+\mathrm{s}_{3, \mathrm{rt}}\right)\right] \text {, que leva a } \\
& \mathrm{UBX}_{\mathrm{S}_{\mathrm{rt}} \mathrm{t}}^{3}=\operatorname{Max}\left[0, \operatorname{Max}\left[0,\left(\mathrm{p}_{1, \mathrm{t}}+\mathrm{s}_{1, \mathrm{rt}}\right)-\left(\mathrm{p}_{2, \mathrm{r}}+\mathrm{s}_{2, \mathrm{rt}}\right)\right]+\left(\mathrm{p}_{2, \mathrm{t}}+\mathrm{s}_{2, \mathrm{rt}}\right)-\left(\mathrm{p}_{3, \mathrm{r}}+\mathrm{s}_{3, \mathrm{rt}}\right)\right] .
\end{aligned}
$$

Observa-se que as variáveis $\left(\mathrm{p}_{1, \mathrm{t}}+\mathrm{s}_{1, \mathrm{rt}}\right)$ e $\left(\mathrm{p}_{2, \mathrm{t}}+\mathrm{s}_{2, \mathrm{rt}}\right)$ contribuem para aumentar o valor de $\operatorname{UBX}_{\mathrm{S}_{\mathrm{rt}} \mathrm{t}}^{3}$, enquanto que $\left(\mathrm{p}_{2, \mathrm{r}}+\mathrm{s}_{2, \mathrm{rt}}\right)$ e $\left(\mathrm{p}_{3, \mathrm{r}}+\mathrm{s}_{3, \mathrm{rt}}\right)$ contribuem para diminuir tal valor. Assim, pode-se escrever que:

$$
\mathrm{UBX}_{\mathrm{S}_{\mathrm{rt}} \mathrm{t}}^{3}=\text { função }\left[+\left(\mathrm{p}_{\mathrm{q}, \mathrm{t}}+\mathrm{s}_{\mathrm{q}, \mathrm{rt}}\right) ;-\left(\mathrm{p}_{\mathrm{q}+1, \mathrm{r}}+\mathrm{s}_{\mathrm{q}+1, \mathrm{rt}}\right) \quad \mathrm{q}=1,2\right] \text {. }
$$

Repetindo o procedimento para $\mathrm{k}=3,4, \ldots, \mathrm{m}-1$, tem-se que na última máquina $\mathrm{m}$,

$$
\mathrm{UBX}_{\mathrm{S}_{\mathrm{rt}} \mathrm{t}}^{\mathrm{m}}=\text { função }\left[+\left(\mathrm{p}_{\mathrm{q}, \mathrm{t}}+\mathrm{s}_{\mathrm{q}, \mathrm{rt}}\right) ;-\left(\mathrm{p}_{\mathrm{q}+1, \mathrm{r}}+\mathrm{s}_{\mathrm{q}+1, \mathrm{rt}}\right) \quad \mathrm{q}=1,2, \ldots, \mathrm{m}-1\right] \text {. }
$$

Desta forma, para os pares de tarefas sucessivas $\left(\mathrm{J}_{\mathrm{t}}, \mathrm{J}_{\mathrm{u}}\right)$ e $\left(\mathrm{J}_{\mathrm{u}}, \mathrm{J}_{\mathrm{v}}\right)$ pode-se escrever:

$$
\begin{aligned}
& \mathrm{UBX}_{\mathrm{S}_{\mathrm{tu}}^{\mathrm{u}}}^{\mathrm{m}}=\text { função }\left[+\left(\mathrm{p}_{\mathrm{q}, \mathrm{u}}+\mathrm{s}_{\mathrm{q}, \mathrm{tu}}\right) ;-\left(\mathrm{p}_{\mathrm{q}+1, \mathrm{t}}+\mathrm{s}_{\mathrm{q}+1, \mathrm{tu}}\right)\right. \\
& \left.\mathrm{UBX}_{\mathrm{S}_{\mathrm{uv}} \mathrm{v}}^{\mathrm{m}}=\text { função }\left[+\left(\mathrm{p}_{\mathrm{q}, \mathrm{v}}+\mathrm{s}_{\mathrm{q}, \mathrm{uv}}\right) ;-\left(\mathrm{p}_{\mathrm{q}+1, \mathrm{u}}+\mathrm{s}_{\mathrm{q}+1, \mathrm{uv}}\right) \quad \mathrm{q}=1,2, \ldots, \mathrm{m}-1\right] \mathrm{e}-1\right] .
\end{aligned}
$$


Nas expressões (28) e (29) nota-se que $\mathrm{UBX}_{\mathrm{S}_{\mathrm{rt}} \mathrm{t}}^{\mathrm{m}}$ e $\mathrm{UBX}_{\mathrm{S}_{\mathrm{tu}} \mathrm{u}}^{\mathrm{m}}$ são dependentes do mesmo conjunto de variáveis $\mathrm{p}_{\mathrm{q}, \mathrm{t}}$ para $\mathrm{q}=2,3, \ldots, \mathrm{m}-1$. Isso indica que tais variáveis aleatórias são dependentes, porém apresentando correlação negativa, uma vez que $\mathrm{p}_{\mathrm{q}, \mathrm{t}}(\mathrm{q}=2,3, \ldots, \mathrm{m}-1)$ contribui para aumentar $\mathrm{UBX}_{\mathrm{S}_{\mathrm{rt}} \mathrm{t}}^{\mathrm{m}}$ e simultaneamente para diminuir $\mathrm{UBX}_{\mathrm{S}_{\mathrm{tu}} \mathrm{u}}^{\mathrm{m}}$. O mesmo acontece com $\mathrm{UBX}_{\mathrm{S}_{\mathrm{tu}} \mathrm{u}}^{\mathrm{m}}$ e $\mathrm{UBX}_{\mathrm{S}_{\mathrm{uv}} \mathrm{v}}^{\mathrm{m}}$, expressões (29) e (30), tendo em vista a dependência simultânea com as variáveis $\mathrm{p}_{\mathrm{q}, \mathrm{u}}(\mathrm{q}=2,3, \ldots, \mathrm{m}-1)$. Entretanto, as variáveis $\mathrm{UBX}_{\mathrm{S}_{\mathrm{rt}}}^{\mathrm{m}} \mathrm{e}$ $\mathrm{UBX}_{\mathrm{S}_{\mathrm{uv}} \mathrm{v}}^{\mathrm{m}}$ são independentes, uma vez que os tempos de processamento das tarefas $\mathrm{J}_{\mathrm{r}}, \mathrm{J}_{\mathrm{t}}$, $\mathrm{J}_{\mathrm{u}}$ e $\mathrm{J}_{\mathrm{V}}$, e os tempos de preparação das máquinas para execução das tarefas $\mathrm{J}_{\mathrm{t}}$ e $\mathrm{J}_{\mathrm{V}}$ são variáveis aleatórias independentes.

Desta forma, conclui-se que as $n$ variáveis $\mathrm{UBX}_{[j+1]}^{\mathrm{m}}, \mathrm{j}=0,1, \ldots, \mathrm{n}-1$, apresentam dependência somente nos pares sucessivos $\left(\mathrm{UBX}_{[j+1]}^{\mathrm{m}}, \mathrm{UBX}_{[j+2]}^{\mathrm{m}}\right), j=0,1, \ldots, n-2$, com covariância negativa.

Utilizando a expressão (23) e as relações de dependência entre as variáveis que fornecem o valor de $\operatorname{UBM}(\sigma)$ na expressão (21), pode-se escrever que:

$$
\begin{aligned}
& \operatorname{var}[\operatorname{UBM}(\sigma)]=\sum_{j=0}^{n-1} \operatorname{var}\left[p_{m[j]}+s_{m[j+1]}\right]+\operatorname{var}\left[p_{m[n]}\right]+\sum_{j=0}^{n-1} \operatorname{var}\left[\operatorname{UBX}_{[j+1]}^{m}\right]+ \\
& +2 \sum_{j=0}^{n-1} \operatorname{cov}\left[\left(p_{m[j]}+s_{m[j+1]}\right), U B X_{[j+1]}^{m}\right]+2 \sum_{j=0}^{n-2} \operatorname{cov}\left[\mathrm{UBX}_{[j+1]}^{m}, \mathrm{UBX}_{[\mathrm{j}+2]}^{\mathrm{m}}\right] .
\end{aligned}
$$

As covariâncias da expressão acima são negativas, portanto

$$
\operatorname{var}[\operatorname{UBM}(\sigma)]<\sum_{\mathrm{j}=0}^{\mathrm{n}-1} \operatorname{var}\left[\mathrm{p}_{\mathrm{m}[\mathrm{j}]}+\mathrm{s}_{\mathrm{m}[\mathrm{j}+1]}\right]+\operatorname{var}\left[\mathrm{p}_{\mathrm{m}[\mathrm{n}]}\right]+\sum_{\mathrm{j}=0}^{\mathrm{n}-1} \operatorname{var}\left[\mathrm{UBX}_{[\mathrm{j}+1]}^{\mathrm{m}}\right] \text {. }
$$

A expressão (20) fornece um limitante superior da variância $\operatorname{var}\left[\mathrm{UBX}_{\mathrm{S}_{\mathrm{uv}}^{\mathrm{k}} \mathrm{v}}^{\mathrm{k}}\right]$ para $\mathrm{k}=1,2, \ldots . ., \mathrm{m}-1$, e qualquer par $\left(\mathrm{J}_{\mathrm{u}}, \mathrm{J}_{\mathrm{v}}\right)$ de tarefas sucessivas. Portanto, para a última máquina $\mathrm{m}$,

$$
\operatorname{var}\left[\mathrm{UBX}_{[\mathrm{j}+1]}^{\mathrm{m}}\right]<2(\mathrm{~m}-1)(\operatorname{var}[\mathrm{P}]+\operatorname{var}[\mathrm{S}]) \quad \forall \mathrm{j}=0,1, \ldots, \mathrm{n}-1 .
$$

Com a expressão (32) e sabendo-se que

$$
\begin{aligned}
& \operatorname{var}\left[\mathrm{p}_{\mathrm{m}[0]}\right]=0, \operatorname{var}\left[\mathrm{s}_{\mathrm{m}[1]}\right]=\operatorname{var}[\mathrm{S}], \operatorname{var}\left[\mathrm{p}_{\mathrm{m}[\mathrm{n}]}\right]=\operatorname{var}[\mathrm{P}], \mathrm{e} \\
& \operatorname{var}\left[\mathrm{p}_{\mathrm{m}[\mathrm{j}]}+\mathrm{s}_{\mathrm{m}[\mathrm{j}+1]}\right]=\operatorname{var}\left[\mathrm{p}_{\mathrm{m}[\mathrm{j}]}\right]+\operatorname{var}\left[\mathrm{s}_{\mathrm{m}[\mathrm{j}+1]}\right]=\operatorname{var}[\mathrm{P}]+\operatorname{var}[\mathrm{S}] \forall \mathrm{j}=1, \ldots, \mathrm{n}-1, \text { obtém-se: }
\end{aligned}
$$




$$
\begin{aligned}
& \operatorname{var}[\mathrm{UBM}(\sigma)]<\mathrm{n}(\operatorname{var}[\mathrm{P}]+\operatorname{var}[\mathrm{S}])+2 \mathrm{n}(\mathrm{m}-1)(\operatorname{var}[\mathrm{P}]+\operatorname{var}[\mathrm{S}]), \text { ou seja, } \\
& \operatorname{var}[\mathrm{UBM}(\sigma)]<\mathrm{n}(2 \mathrm{~m}-1)(\operatorname{var}[\mathrm{P}]+\operatorname{var}[\mathrm{S}]) .
\end{aligned}
$$

Assim, a expressão (33) fornece um limitante superior da variância de $\operatorname{UBM}(\sigma)$, conforme segue:

$\operatorname{LS}(\operatorname{var}[\operatorname{UBM}(\sigma)])=\mathrm{n}(2 \mathrm{~m}-1)(\operatorname{var}[\mathrm{P}]+\operatorname{var}[\mathrm{S}])$.

\subsubsection{Variável $\mathrm{M}(\sigma)$}

Por definição,

$$
M(\sigma)=\sum_{j=1}^{n} p_{m[j]}+\sum_{j=0}^{n-1} s_{m[j+1]}+\sum_{j=0}^{n-1} X_{[j+1]}^{m} \leq \operatorname{UBM}(\sigma)=\sum_{j=1}^{n} p_{m[j]}+\sum_{j=0}^{n-1} s_{m[j+1]}+\sum_{j=0}^{n-1} U B X_{[j+1]}^{m}
$$

Desta forma, os limitantes associados à variável $\operatorname{UBM}(\sigma)$ podem ser adotados como limitantes do makespan $\mathrm{M}(\sigma)$.

\subsubsection{Variável Desvio Relativo $\operatorname{DR}(\sigma)$}

Por definição,

$$
\operatorname{DR}(\sigma)=\frac{\mathrm{UBM}(\sigma)-\mathrm{M}(\sigma)}{\mathrm{M}(\sigma)} \text {, ou seja, } \operatorname{DR}(\sigma)=\frac{\mathrm{UBM}(\sigma)}{\mathrm{M}(\sigma)}-1 .
$$

Assim,

$$
\operatorname{var}[\operatorname{DR}(\sigma)]=\operatorname{var}\left[\frac{\operatorname{UBM}(\sigma)}{\mathrm{M}(\sigma)}\right] \text {. }
$$

Sabe-se que se $\mathrm{Z}$ e $\mathrm{W}$ forem duas variáveis aleatórias, então a variância $\operatorname{var}\left[\frac{\mathrm{Z}}{\mathrm{W}}\right]$ é aproximadamente calculada pela expressão:

$$
\operatorname{var}\left[\frac{\mathrm{Z}}{\mathrm{W}}\right] \approx\left(\frac{\mu[\mathrm{Z}]}{\mu[\mathrm{W}]}\right)^{2}\left(\frac{\operatorname{var}[\mathrm{Z}]}{\mu[\mathrm{Z}]^{2}}+\frac{\operatorname{var}[\mathrm{W}]}{\mu[\mathrm{W}]^{2}}-\frac{2 \operatorname{cov}[\mathrm{Z}, \mathrm{W}]}{\mu[\mathrm{Z}] \mu[\mathrm{W}]}\right) .
$$

Fazendo $\operatorname{UBM}(\sigma)=\mathrm{Z}$ e $\mathrm{M}(\sigma)=\mathrm{W}$, obtém-se

$$
\operatorname{var}\left[\frac{\operatorname{UBM}(\sigma)}{\mathrm{M}(\sigma)}\right] \approx\left(\frac{\mu[\operatorname{UBM}(\sigma)]}{\mu[\mathrm{M}(\sigma)]}\right)^{2}\left(\frac{\operatorname{var}[\operatorname{UBM}(\sigma)]}{\mu[\operatorname{UBM}(\sigma)]^{2}}+\frac{\operatorname{var}[\mathrm{M}(\sigma)]}{\mu[\mathrm{M}(\sigma)]^{2}}-\frac{2 \operatorname{cov}[\operatorname{UBM}(\sigma), \mathrm{M}(\sigma)]}{\mu[\operatorname{UBM}(\sigma)] \mu[\mathrm{M}(\sigma)]}\right)
$$


Pode-se notar, pelas expressões (14) e (15), que $\operatorname{UBM}(\sigma)$ e $\mathrm{M}(\sigma)$ são variáveis aleatórias dependentes e com correlação positiva, portanto apresentando covariância positiva. Assim, a partir da expressão (36) pode-se escrever que:

$\operatorname{var}\left[\frac{\operatorname{UBM}(\sigma)}{\mathrm{M}(\sigma)}\right]<\left(\frac{\mu[\operatorname{UBM}(\sigma)]}{\mu[\mathrm{M}(\sigma)]}\right)^{2}\left(\frac{\operatorname{var}[\operatorname{UBM}(\sigma)]}{\mu[\operatorname{UBM}(\sigma)]^{2}}+\frac{\operatorname{var}[\mathrm{M}(\sigma)]}{\mu[\mathrm{M}(\sigma)]^{2}}\right)$.

Substituindo adequadamente na expressão (37) as médias e variâncias de $\operatorname{UBM}(\sigma)$ e $\mathrm{M}(\sigma)$ pelos correspondentes limitantes superiores e inferiores, tem-se que:

$\operatorname{var}\left[\frac{\operatorname{UBM}(\sigma)}{\mathrm{M}(\sigma)}\right]<\left(\frac{\operatorname{LS}(\mu[\operatorname{UBM}(\sigma)])}{\operatorname{LI}(\mu[\mathrm{M}(\sigma)])}\right)^{2}\left(\frac{\operatorname{LS}(\operatorname{var}[\operatorname{UBM}(\sigma)])}{\operatorname{LI}(\mu[\operatorname{UBM}(\sigma)])^{2}}+\frac{\operatorname{LS}(\operatorname{var}[\mathrm{M}(\sigma)])}{\operatorname{LI}(\mu[\mathrm{M}(\sigma)])^{2}}\right)$, onde

(26) $\operatorname{LS}(\mu[\operatorname{UBM}(\sigma)])=\mathrm{n}(\mu[\mathrm{P}]+\mu[\mathrm{S}]+(\mathrm{m}-1)(\mathrm{MAX}[\mathrm{P}+\mathrm{S}]-\mathrm{MIN}[\mathrm{P}+\mathrm{S}]))$;

(27) LI $(\mu[\operatorname{UBM}(\sigma)])=\mathrm{n}(\mu[\mathrm{P}]+\mu[\mathrm{S}])$, sendo que LI $(\mu[\mathrm{M}(\sigma)])=\operatorname{LI}(\mu[\operatorname{UBM}(\sigma)])$;

(34) LS $(\operatorname{var}[\operatorname{UBM}(\sigma)])=\mathrm{n}(2 \mathrm{~m}-1)(\operatorname{var}[\mathrm{P}]+\operatorname{var}[\mathrm{S}])$, sendo que LS $(\operatorname{var}[\mathrm{M}(\sigma)])=\mathrm{LS}(\operatorname{var}[\operatorname{UBM}(\sigma)])$.

Desta forma,

$$
\begin{aligned}
& \operatorname{var}\left[\frac{\operatorname{UBM}(\sigma)}{\mathrm{M}(\sigma)}\right]<\left(\frac{\operatorname{LS}(\mu[\operatorname{UBM}(\sigma)])}{\operatorname{LI}(\mu[\mathrm{UBM}(\sigma)])}\right)^{2}\left(2 \frac{\operatorname{LS}(\operatorname{var}[\mathrm{UBM}(\sigma)])}{\operatorname{LI}(\mu[\operatorname{UBM}(\sigma)])^{2}}\right), \text { ou seja, } \\
& \operatorname{var}\left[\frac{\mathrm{UBM}(\sigma)}{\mathrm{M}(\sigma)}\right]<2 \mathrm{LS}(\operatorname{var}[\operatorname{UBM}(\sigma)])\left(\frac{\mathrm{LS}(\mu[\mathrm{UBM}(\sigma)])^{2}}{\operatorname{LI}(\mu[\mathrm{UBM}(\sigma)])^{4}}\right), \text { que leva a } \\
& \operatorname{var}\left[\frac{\mathrm{UBM}(\sigma)}{\mathrm{M}(\sigma)}\right]<2 \mathrm{n}(2 \mathrm{~m}-1)(\operatorname{var}[\mathrm{P}]+\operatorname{var}[\mathrm{S}])\left(\frac{\mathrm{n}^{2}(\mu[\mathrm{P}]+\mu[\mathrm{S}]+(\mathrm{m}-1)(\mathrm{MAX}[\mathrm{P}+\mathrm{S}]-\mathrm{MIN}[\mathrm{P}+\mathrm{S}]))^{2}}{\mathrm{n}^{4}(\mu[\mathrm{P}]+\mu[\mathrm{S}])^{4}}\right)
\end{aligned}
$$

Portanto,

$$
\operatorname{var}\left[\frac{\mathrm{UBM}(\sigma)}{\mathrm{M}(\sigma)}\right]<\frac{2(2 \mathrm{~m}-1)(\operatorname{var}[\mathrm{P}]+\operatorname{var}[\mathrm{S}])(\mu[\mathrm{P}]+\mu[\mathrm{S}]+(\mathrm{m}-1)(\mathrm{MAX}[\mathrm{P}+\mathrm{S}]-\mathrm{MIN}[\mathrm{P}+\mathrm{S}]))^{2}}{\mathrm{n}(\mu[\mathrm{P}]+\mu[\mathrm{S}])^{4}} .
$$

A expressão (38) fornece um limitante superior da $\operatorname{var}[\operatorname{DR}(\sigma)]=\operatorname{var}\left[\frac{\mathrm{UBM}(\sigma)}{\mathrm{M}(\sigma)}\right]$. Conseqüentemente, também fornece um limitante superior do desvio padrão $S_{\mathrm{DR}}$ dado por:

$\operatorname{LS}\left(\mathrm{S}_{\mathrm{DR}}\right)=\frac{(\mu[\mathrm{P}]+\mu[\mathrm{S}]+(\mathrm{m}-1)(\mathrm{MAX}[\mathrm{P}+\mathrm{S}]-\mathrm{MIN}[\mathrm{P}+\mathrm{S}]))}{(\mu[\mathrm{P}]+\mu[\mathrm{S}])^{2}} \sqrt{\frac{2(2 \mathrm{~m}-1)(\operatorname{var}[\mathrm{P}]+\operatorname{var}[\mathrm{S}])}{\mathrm{n}}}$. 
Mesmo sem uma avaliação numérica, a expressão (39) possibilita inferir que:

- O grau de equivalência entre os espaços de soluções do problema de programação da produção e do caixeiro-viajante análogo tende a melhorar à medida que aumenta o porte do problema em termos do número $\mathrm{n}$ de tarefas;

- Entretanto, esse grau deve diminuir para um maior número m de máquinas;

- A adequação da analogia entre os mencionados problemas e, portanto o grau de equivalência entre os espaços de soluções apresenta uma tendência de ser melhor quanto maiores forem os tempos $\mathrm{P}$ de processamento das tarefas juntamente com os tempos $\mathrm{S}$ de preparação das máquinas, mais especificamente, quanto maior for o valor de $(\mu[\mathrm{P}]+\mu[\mathrm{S}])$.

\subsection{Experimentação Computacional}

A avaliação numérica do grau de equivalência entre os espaços de soluções do problema de programação da produção e do problema análogo do caixeiro-viajante foi efetuada por meio de uma experimentação computacional descrita a seguir.

Considerou-se um conjunto de problemas com o número de máquinas $\mathrm{m} \varepsilon\{4,7\}$ e o número de tarefas $\mathrm{n} \varepsilon\{10,20,30,40,50,60,70,80,90\}$. Foram utilizadas seis relações para os tempos de processamento das tarefas e os tempos de preparação das máquinas conforme indica a Tabela 1. As relações foram definidas com base em trabalhos reportados na literatura, tais como Simons Jr. (1992), Das, Gupta \& Khumawala (1995), Rajendran \& Ziegler (1997), Rios-Mercado \& Bard (1998, 1999b), e Weng, Lu \& Ren (2001). Os tempos de processamento $(\mathrm{P})$ e os tempos de preparação $(\mathrm{S})$ foram considerados como variáveis aleatórias independentes com distribuição uniforme discreta.

Tabela 1 - Tempos de processamento (P) e tempos de preparação (S).

\begin{tabular}{ccc}
\hline Relação & Intervalo de $\mathbf{P}$ & Intervalo de $\mathbf{S}$ \\
\hline I & $1-99$ & $1-99$ \\
II & $1-99$ & $100-120$ \\
III & $10-99$ & $1-9$ \\
IV & $50-99$ & $1-49$ \\
V & $1-99$ & $1-120$ \\
VI & $1-99$ & $1-20$ \\
\hline
\end{tabular}

Sabe-se que se $\mathrm{Z}$ for uma variável aleatória discreta com distribuição uniforme no intervalo $[a, b]$, então a média $\mu[Z]=\frac{a+b}{2}$ e a variância $\operatorname{var}[Z]=\frac{(b-a+1)^{2}-1}{12}$. A partir das distribuições de P e S da Tabela 1 e utilizando-se a expressão (39) podem ser obtidos os valores dos limitantes superiores do Desvio Padrão $\mathrm{S}_{\mathrm{DR}}$ conforme apresentados na Tabela 2. 
Tabela 2 - Limitantes superiores LS ( $\left.\mathrm{S}_{\mathrm{DR}}\right)$ em função do número n de tarefas.

\begin{tabular}{|c|c|c|c|c|c|c|}
\hline \multirow{3}{*}{$\begin{array}{l}\text { Número } \\
\text { de } \\
\text { máquinas }\end{array}$} & \multicolumn{6}{|c|}{$\mathrm{LS}\left(\mathrm{S}_{\mathrm{DR}}\right)$} \\
\hline & \multicolumn{6}{|c|}{ Relações de P e S } \\
\hline & I & II & III & IV & $\mathbf{V}$ & VI \\
\hline$m=4$ & $10,4 / \sqrt{n}$ & $2,2 / \sqrt{n}$ & $9,7 / \sqrt{n}$ & $3,0 / \sqrt{n}$ & $10,5 / \sqrt{\mathrm{n}}$ & $12,3 / \sqrt{n}$ \\
\hline$m=7$ & $26,3 / \sqrt{n}$ & $5,1 / \sqrt{\mathrm{n}}$ & $24,1 / \sqrt{\mathrm{n}}$ & $7,1 / \sqrt{\mathrm{n}}$ & $26,5 / \sqrt{n}$ & $31,0 / \sqrt{\mathrm{n}}$ \\
\hline
\end{tabular}

A Tabela 2 indica que, em termos relativos, as Relações II e IV devem levar aos menores valores do Desvio Padrão $\mathrm{S}_{\mathrm{DR}}$.

$\mathrm{Na}$ experimentação, para cada classe $(\mathrm{m} X \mathrm{n})$ de problemas foi gerada aleatoriamente uma seqüência-semente inicial. A partir dessa seqüência-semente, foram obtidas todas as seqüências da respectiva Vizinhança de Inserção, constituída de $(n-1)^{2}$ seqüências. A Vizinhança de Inserção é obtida pela mudança de posição de cada uma das n tarefas para as (n-1) posições diferentes na seqüência-semente. Para cada seqüência vizinha $\sigma$ calculou-se o respectivo valor do Desvio Relativo $\operatorname{DR}(\sigma)$. Tendo os valores dos desvios relativos, foram calculados os valores do Desvio Relativo Médio $\overline{\mathrm{DR}}$ e do Desvio Padrão do desvio relativo $\mathrm{S}_{\mathrm{DR}}$ correspondentes à Vizinhança da seqüência-semente.

Com o objetivo de melhor estimar os valores de $\overline{\mathrm{DR}}$ e $\mathrm{S}_{\mathrm{DR}}$ para o espaço de soluções (n! seqüências), uma segunda seqüência-semente foi gerada da seguinte forma: dividiu-se aleatoriamente a última seqüência vizinha da seqüência-semente inicial em duas partes, invertendo-se as posições de todas as tarefas que compõem essas duas partes. Desta forma, procurou-se explorar melhor o espaço de soluções com um adequado grau de diversidade. Para a Vizinhança de Inserção da nova seqüência-semente, foram calculados os respectivos valores do Desvio Relativo Médio $\overline{\mathrm{DR}}$ e do Desvio Padrão do desvio relativo $\mathrm{S}_{\mathrm{DR}}$, os quais juntamente com os correspondentes à primeira seqüência-semente foram utilizados para $\mathrm{o}$ cálculo de $\overline{\mathrm{DR}}$ e $\mathrm{S}_{\mathrm{DR}}$ correspondentes ao conjunto de $2(\mathrm{n}-1)^{2}$ seqüências, envolvendo portanto as duas vizinhanças geradas.

Esse procedimento foi repetido sucessivamente até se atingir um valor estável para o Desvio Padrão do desvio relativo $\mathrm{S}_{\mathrm{DR}}$ correspondente ao conjunto total das seqüências (soluções viáveis) até então avaliadas. Considerou-se que o Desvio Padrão $\mathrm{S}_{\mathrm{DR}}$ atingiu a estabilidade quando seu valor permaneceu inalterado para 1000 seqüências-semente sucessivas, com uma precisão de $0,001(0,1 \%)$. Isso significa que, para cada classe $(\mathrm{m} X \mathrm{n})$ de problemas foram avaliadas, no mínimo, $1000(\mathrm{n}-1)^{2}$ seqüências.

Os resultados obtidos na experimentação computacional são apresentados nas Tabelas 3 e 4 . Os valores $(N s)$ indicam o número de seqüências-semente geradas para a estabilização do Desvio Padrão $\mathrm{S}_{\mathrm{DR}}$. Nas Tabelas, os melhores resultados (menores $\mathrm{S}_{\mathrm{DR}}$ ) estão salientados em negrito e os maiores $S_{D R}$ grifados. 
Tabela 3 - Resultados para tempos de preparação das máquinas independentes da seqüência das tarefas.

\begin{tabular}{|c|c|c|c|c|c|c|c|c|c|c|c|c|c|}
\hline \multirow{4}{*}{\multicolumn{2}{|c|}{$\begin{array}{l}\text { Class } \\
\text { m X n }\end{array}$}} & \multicolumn{12}{|c|}{ Relações de P e S } \\
\hline & & \multicolumn{2}{|r|}{ I } & \multicolumn{2}{|c|}{ II } & \multicolumn{2}{|c|}{ III } & \multicolumn{2}{|c|}{ IV } & \multicolumn{2}{|c|}{$\mathbf{V}$} & \multicolumn{2}{|c|}{ VI } \\
\hline & & \multicolumn{12}{|c|}{ Valores em porcentagem $(\%)$} \\
\hline & & $\overline{\mathbf{D R}}$ & $\begin{array}{c}S_{\text {DR }} \\
(N s) \\
\end{array}$ & $\overline{\mathrm{DR}}$ & $\begin{array}{l}S_{\text {DR }} \\
(N s) \\
\end{array}$ & $\overline{\mathrm{DR}}$ & $\begin{array}{l}\mathrm{S}_{\mathrm{DR}} \\
(\mathrm{Ns}) \\
\end{array}$ & $\overline{\mathbf{D R}}$ & $\begin{array}{c}\mathrm{S}_{\mathrm{DR}} \\
(\mathrm{Ns}) \\
\end{array}$ & $\overline{\mathbf{D R}}$ & $\begin{array}{c}\mathrm{S}_{\mathrm{DR}} \\
(\mathrm{Ns}) \\
\end{array}$ & $\overline{\mathbf{D R}}$ & $\begin{array}{l}\mathrm{S}_{\mathrm{DR}} \\
(\mathrm{Ns}) \\
\end{array}$ \\
\hline \multirow{9}{*}{4} & 10 & 16,2 & $\frac{2,9}{(2210)}$ & 7,9 & $\begin{array}{c}\mathbf{1 , 4} \\
(1531)\end{array}$ & 16,8 & \begin{tabular}{c|}
2,8 \\
$(1575)$
\end{tabular} & 9,9 & $\begin{array}{c}1,5 \\
(1788)\end{array}$ & 15,2 & $\begin{array}{c}2,4 \\
(2174) \\
\end{array}$ & 20,6 & $\frac{2,9}{(1638)}$ \\
\hline & 20 & 23,8 & $\begin{array}{c}1,8 \\
(2204) \\
\end{array}$ & 11,2 & \begin{tabular}{|c|}
$\mathbf{0 , 8}$ \\
$(1533)$ \\
\end{tabular} & 23,5 & \begin{tabular}{|c|}
1,7 \\
$(2103)$ \\
\end{tabular} & 11,5 & \begin{tabular}{|c|}
$\mathbf{0 , 8}$ \\
$(1976)$ \\
\end{tabular} & 22,0 & $\begin{array}{c}1,5 \\
(1435) \\
\end{array}$ & 29,1 & $\frac{2,0}{(1884)}$ \\
\hline & 30 & 25,2 & $\begin{array}{c}1,2 \\
(1567) \\
\end{array}$ & 14,8 & \begin{tabular}{|c|}
$\mathbf{0 , 6}$ \\
$(2107)$ \\
\end{tabular} & 28,3 & \begin{tabular}{c|}
1,4 \\
$(1857)$ \\
\end{tabular} & 13,5 & $\begin{array}{c}\mathbf{0 , 6} \\
(1661) \\
\end{array}$ & 27,2 & $\begin{array}{c}1,2 \\
(1254) \\
\end{array}$ & 35,1 & $(1,89)$ \\
\hline & 40 & 28,6 & $\begin{array}{c}1,0 \\
(1297) \\
\end{array}$ & 15,3 & $\begin{array}{c}\mathbf{0 , 5} \\
(1477) \\
\end{array}$ & 33,2 & \begin{tabular}{c|}
1,1 \\
$(1866)$
\end{tabular} & 14,9 & $\begin{array}{c}\mathbf{0 , 5} \\
(1314) \\
\end{array}$ & 28,6 & $\begin{array}{c}1,0 \\
(1147) \\
\end{array}$ & 40,1 & $(1,449)$ \\
\hline & 50 & 30,1 & $\begin{array}{c}0,7 \\
(1535) \\
\end{array}$ & 16,2 & $\begin{array}{c}\mathbf{0 , 4} \\
(1055)\end{array}$ & 36,8 & \begin{tabular}{c|}
1,0 \\
$(1175)$
\end{tabular} & 15,9 & $\begin{array}{c}\mathbf{0 , 4} \\
(1105)\end{array}$ & 29,2 & \begin{tabular}{c|}
0,7 \\
$(1737)$
\end{tabular} & 41,3 & $\frac{1,1}{(2041)}$ \\
\hline & 60 & 30,2 & $\begin{array}{c}0,6 \\
(1680)\end{array}$ & 16,7 & $\begin{array}{c}0,4 \\
(1005)\end{array}$ & 38,9 & $\frac{0,9}{(1145)}$ & 16,9 & $\begin{array}{c}\mathbf{0 , 3} \\
(1129)\end{array}$ & 30,6 & $\begin{array}{c}0,6 \\
(1452) \\
\end{array}$ & 41,9 & $\left(\frac{0,9}{2075)}\right.$ \\
\hline & 70 & 30,5 & \begin{tabular}{|c|}
0,6 \\
$(1632)$ \\
\end{tabular} & 16,7 & \begin{tabular}{|c|}
$\mathbf{0 , 3}$ \\
$(1005)$ \\
\end{tabular} & 39,3 & \begin{tabular}{|c|}
0,8 \\
$(1225)$ \\
\end{tabular} & 17,1 & \begin{tabular}{|c|}
$\mathbf{0 , 3}$ \\
$(1016)$ \\
\end{tabular} & 32,6 & $\begin{array}{c}0,6 \\
(1403) \\
\end{array}$ & 43,8 & $(\underline{0,9})$ \\
\hline & 80 & 32,9 & $\begin{array}{c}0,5 \\
(1283) \\
\end{array}$ & 16,7 & $\begin{array}{c}\mathbf{0 , 3} \\
(1039) \\
\end{array}$ & 39,7 & $\frac{0,7}{(1458)}$ & 17,6 & $\begin{array}{c}\mathbf{0 , 3} \\
(1001) \\
\end{array}$ & 34,7 & $\begin{array}{c}0,5 \\
(1267) \\
\end{array}$ & 44,0 & $\left(\frac{0,7}{(1043)}\right.$ \\
\hline & 90 & 34,2 & $\begin{array}{c}0,5 \\
(1764) \\
\end{array}$ & 18,4 & \begin{tabular}{|c|}
$\mathbf{0 , 2}$ \\
$(1797)$ \\
\end{tabular} & 42,2 & \begin{tabular}{|c|}
$\underline{0,6}$ \\
$(1264)$ \\
\end{tabular} & 18,1 & $\begin{array}{c}\mathbf{0 , 2} \\
(1026) \\
\end{array}$ & 34,8 & $\begin{array}{c}0,4 \\
(1504) \\
\end{array}$ & 45,6 & $\underline{0,6}$ \\
\hline & & & & & & & & & & & & & \\
\hline \multirow{9}{*}{7} & 10 & 19,1 & \begin{tabular}{|c|}
2,6 \\
$(1965)$ \\
\end{tabular} & 10,9 & \begin{tabular}{c|}
1,6 \\
$(2107)$
\end{tabular} & 17,4 & \begin{tabular}{|c|}
2,6 \\
$(2185)$ \\
\end{tabular} & 11,1 & $\begin{array}{c}\mathbf{1 , 5} \\
(1644)\end{array}$ & 17,2 & \begin{tabular}{|c|}
2,7 \\
$(2028)$
\end{tabular} & 22,0 & $\frac{2,9}{(2218)}$ \\
\hline & 20 & 25,9 & \begin{tabular}{|c|}
1,8 \\
$(1970)$ \\
\end{tabular} & 19,5 & $\begin{array}{c}1,2 \\
(2610) \\
\end{array}$ & 34,1 & \begin{tabular}{|c|}
2,3 \\
$(1939)$ \\
\end{tabular} & 17,1 & $\begin{array}{c}\mathbf{1 , 1} \\
(1136) \\
\end{array}$ & 27,8 & $\begin{array}{c}1,8 \\
(1473) \\
\end{array}$ & 38,4 & $\frac{2,5}{(2021)}$ \\
\hline & 30 & 31,7 & \begin{tabular}{c|}
1,3 \\
$(1875)$ \\
\end{tabular} & 23,2 & $\begin{array}{c}0,9 \\
(1688)\end{array}$ & 41,8 & \begin{tabular}{|c|}
1,8 \\
$(1268)$ \\
\end{tabular} & 18,7 & $\begin{array}{c}\mathbf{0 , 8} \\
(1115)\end{array}$ & 31,8 & $\begin{array}{c}1,3 \\
(1656)\end{array}$ & 45,9 & $(11,9)$ \\
\hline & 40 & 38,9 & $\begin{array}{c}1,1 \\
(2135)\end{array}$ & 25,0 & $\begin{array}{c}0,7 \\
(1423)\end{array}$ & 46,8 & $\frac{1,6}{(1273)}$ & 21,4 & $\begin{array}{c}\mathbf{0 , 6} \\
(1620) \\
\end{array}$ & 36,0 & $\begin{array}{c}1,1 \\
(1952) \\
\end{array}$ & 47,2 & $\left(\frac{1,6}{(1139)}\right.$ \\
\hline & 50 & 42,1 & \begin{tabular}{|c|}
1,0 \\
$(1766)$ \\
\end{tabular} & 25,3 & \begin{tabular}{|c|}
$\mathbf{0 , 5}$ \\
$(1758)$ \\
\end{tabular} & 50,0 & \begin{tabular}{|c|}
1,2 \\
$(1879)$ \\
\end{tabular} & 21,9 & $\begin{array}{c}\mathbf{0 , 5} \\
(1330)\end{array}$ & 39,8 & $\begin{array}{c}1,0 \\
(1258)\end{array}$ & 52,9 & $\frac{1,3}{(1489)}$ \\
\hline & 60 & 46,4 & $\begin{array}{c}0,9 \\
(1716) \\
\end{array}$ & 26,3 & $\begin{array}{c}0,5 \\
(1089)\end{array}$ & 54,6 & \begin{tabular}{c|}
1,1 \\
$(1420)$ \\
\end{tabular} & 22,4 & $\begin{array}{c}\mathbf{0 , 4} \\
(1632) \\
\end{array}$ & 41,2 & $\begin{array}{c}0,8 \\
(1158)\end{array}$ & 55,7 & $\left(\frac{1,2}{1151)}\right.$ \\
\hline & 70 & 47,0 & $\begin{array}{c}0,8 \\
(1277) \\
\end{array}$ & 26,6 & $\begin{array}{c}\mathbf{0 , 4} \\
(1450)\end{array}$ & 54,8 & \begin{tabular}{|c|}
0,9 \\
$(2023)$ \\
\end{tabular} & 24,8 & $\begin{array}{c}\mathbf{0 , 4} \\
(1010) \\
\end{array}$ & 42,7 & $\begin{array}{c}0,7 \\
(1339)\end{array}$ & 58,3 & $\left(\frac{1,1}{(1330)}\right.$ \\
\hline & 80 & 47,7 & \begin{tabular}{|c|}
0,7 \\
$(1374)$
\end{tabular} & 26,7 & $\begin{array}{c}0,4 \\
(1043)\end{array}$ & 59,7 & $\frac{0,9}{(1177)}$ & 25,6 & \begin{tabular}{|c|}
$\mathbf{0 , 3}$ \\
$(1276)$
\end{tabular} & 42,7 & $\begin{array}{c}0,6 \\
(1083)\end{array}$ & 59,8 & $\frac{0,9}{(1889)}$ \\
\hline & 90 & 47,6 & \begin{tabular}{|c|}
0,6 \\
$(1699)$
\end{tabular} & 27,5 & $\begin{array}{c}\mathbf{0 , 3} \\
(1196)\end{array}$ & 62,5 & $\frac{0,8}{(1736)}$ & 27,7 & $\begin{array}{c}\mathbf{0 , 3} \\
(1102)\end{array}$ & 47,9 & $\begin{array}{c}0,6 \\
(1246)\end{array}$ & 64,7 & $\left(\frac{0,8}{1953)}\right.$ \\
\hline
\end{tabular}


Tabela 4 - Resultados para tempos de preparação das máquinas dependentes da seqüência das tarefas.

\begin{tabular}{|c|c|c|c|c|c|c|c|c|c|c|c|c|c|}
\hline \multirow{4}{*}{\multicolumn{2}{|c|}{$\begin{array}{l}\text { Classe } \\
\text { m X n }\end{array}$}} & \multicolumn{12}{|c|}{ Relações de P e S } \\
\hline & & \multicolumn{2}{|r|}{ I } & \multicolumn{2}{|c|}{ II } & \multicolumn{2}{|c|}{ III } & \multicolumn{2}{|c|}{ IV } & \multicolumn{2}{|c|}{$\mathbf{V}$} & \multicolumn{2}{|c|}{ VI } \\
\hline & & \multicolumn{12}{|c|}{ Valores em porcentagem (\%) } \\
\hline & & $\overline{\mathrm{DR}}$ & $\begin{array}{l}S_{\text {DR }} \\
(N s) \\
\end{array}$ & $\overline{\mathrm{DR}}$ & $\begin{array}{l}S_{\text {DR }} \\
(N s) \\
\end{array}$ & $\overline{\mathrm{DR}}$ & $\begin{array}{l}\mathrm{S}_{\mathrm{DR}} \\
(\mathrm{Ns}) \\
\end{array}$ & $\overline{\mathrm{DR}}$ & $\begin{array}{l}\mathrm{S}_{\mathrm{DR}} \\
(\mathrm{Ns}) \\
\end{array}$ & $\overline{\mathrm{DR}}$ & $\begin{array}{l}\mathrm{S}_{\mathrm{DR}} \\
(\mathrm{Ns}) \\
\end{array}$ & $\overline{\mathrm{DR}}$ & $\begin{array}{l}\mathrm{S}_{\mathrm{DR}} \\
(N s) \\
\end{array}$ \\
\hline \multirow{9}{*}{4} & 10 & 19,0 & $\begin{array}{c}2,7 \\
(3434)\end{array}$ & 8,1 & $\begin{array}{c}\mathbf{1 , 5} \\
(1387)\end{array}$ & 17,0 & $\begin{array}{c}2,6 \\
(1673)\end{array}$ & 9,0 & $\begin{array}{c}\mathbf{1 , 5} \\
(1543)\end{array}$ & 16,8 & $\begin{array}{c}2,8 \\
(2452)\end{array}$ & 18,5 & $\frac{3,2}{(2054)}$ \\
\hline & 20 & 22,8 & $\begin{array}{c}2,0 \\
(1382)\end{array}$ & 13,8 & $\begin{array}{c}\mathbf{0 , 9} \\
(2163) \\
\end{array}$ & 28,1 & $\begin{array}{c}2,0 \\
(2438) \\
\end{array}$ & 12,4 & $\begin{array}{c}1,0 \\
(1446) \\
\end{array}$ & 23,8 & $\begin{array}{c}2,1 \\
(1976) \\
\end{array}$ & 29,5 & $\frac{2,3}{(2073)}$ \\
\hline & 30 & 27,7 & $\left(\frac{1,6}{(1427)}\right.$ & 14,2 & $\begin{array}{c}\mathbf{0 , 6} \\
(1652) \\
\end{array}$ & 30,1 & $\begin{array}{c}1,5 \\
(1992) \\
\end{array}$ & 13,9 & $\begin{array}{c}0,8 \\
(1542) \\
\end{array}$ & 24,9 & $\left(\frac{1,6}{(1584)}\right.$ & 35,8 & $(2361)$ \\
\hline & 40 & 29,0 & $\left(\frac{1,3}{1933)}\right.$ & 15,3 & \begin{tabular}{|c|}
$\mathbf{0 , 5}$ \\
$(1237)$ \\
\end{tabular} & 34,5 & $\frac{1,3}{(1671)}$ & 14,7 & $\begin{array}{c}0,6 \\
(1493) \\
\end{array}$ & 25,6 & $\left(\frac{1,3}{(1493)}\right.$ & 36,3 & $(2084)$ \\
\hline & 50 & 29,6 & $\frac{1,1}{(2033)}$ & 16,6 & $\begin{array}{c}\mathbf{0 , 4} \\
(1568)\end{array}$ & 36,6 & $\begin{array}{c}1,0 \\
(1230)\end{array}$ & 15,1 & $\begin{array}{c}0,5 \\
(1064) \\
\end{array}$ & 29,3 & $\frac{1,1}{(2127)}$ & 36,3 & $\frac{1,1}{(1446)}$ \\
\hline & 60 & 32,3 & $\left(\frac{1,0}{(1524)}\right.$ & 16,6 & $\begin{array}{c}\mathbf{0 , 4} \\
(1024)\end{array}$ & 38,9 & $\begin{array}{c}0,9 \\
(1915)\end{array}$ & 16,0 & $\begin{array}{c}0,5 \\
(1056)\end{array}$ & 30,1 & $\left(\frac{1,0}{(1724)}\right.$ & 38,2 & $\begin{array}{c}0,9 \\
(1271)\end{array}$ \\
\hline & 70 & 32,4 & $(\underline{0,9})$ & 17,4 & \begin{tabular}{|c|}
$\mathbf{0 , 3}$ \\
$(1106)$ \\
\end{tabular} & 39,6 & $\begin{array}{c}0,8 \\
(1766) \\
\end{array}$ & 16,7 & $\begin{array}{c}0,4 \\
(1159) \\
\end{array}$ & 31,8 & $(\underline{0,9})$ & 39,0 & $\begin{array}{c}0,8 \\
(1216) \\
\end{array}$ \\
\hline & 80 & 33,1 & $\left(\frac{0,8}{1540)}\right.$ & 18,7 & \begin{tabular}{|c|}
$\mathbf{0 , 3}$ \\
$(1099)$ \\
\end{tabular} & 42,0 & \begin{tabular}{|c|}
0,7 \\
$(1922)$ \\
\end{tabular} & 16,8 & $\begin{array}{c}0,4 \\
(1015) \\
\end{array}$ & 32,2 & $\left(\frac{0,8}{1792)}\right.$ & 39,4 & $\begin{array}{c}0,7 \\
(1476)\end{array}$ \\
\hline & 90 & 34,4 & $\frac{0,7}{(1966)}$ & 20,5 & \begin{tabular}{|c|}
$\mathbf{0 , 3}$ \\
$(1017)$ \\
\end{tabular} & 45,8 & $\frac{0,7}{(1290)}$ & 18,3 & \begin{tabular}{|c|}
$\mathbf{0 , 3}$ \\
$(1529)$ \\
\end{tabular} & 33,9 & $\frac{0,7}{(1578)}$ & 43,6 & $\frac{0,7}{(1788)}$ \\
\hline & & & & & & & & & & & & & \\
\hline \multirow{9}{*}{7} & 10 & 23,0 & \begin{tabular}{|c|}
3,1 \\
$(2051)$ \\
\end{tabular} & 12,9 & $\begin{array}{c}1,7 \\
(1243) \\
\end{array}$ & 21,4 & \begin{tabular}{|c|}
3,0 \\
$(2198)$
\end{tabular} & 10,9 & \begin{tabular}{|c|}
$\mathbf{1 , 6}$ \\
$(2015)$ \\
\end{tabular} & 16,7 & \begin{tabular}{|c|}
3,0 \\
$(2397)$ \\
\end{tabular} & 27,6 & $\frac{3,2}{(2683)}$ \\
\hline & 20 & 30,7 & $\begin{array}{c}2,2 \\
(2082) \\
\end{array}$ & 19,5 & $\begin{array}{c}1,2 \\
(1458) \\
\end{array}$ & 33,2 & $\begin{array}{c}2,2 \\
(1715) \\
\end{array}$ & 16,2 & $\begin{array}{c}\mathbf{1 , 1} \\
(1929) \\
\end{array}$ & 26,6 & $\begin{array}{c}2,1 \\
(1501) \\
\end{array}$ & 36,0 & $(2,457)$ \\
\hline & 30 & 33,7 & $\begin{array}{c}1,7 \\
(1457)\end{array}$ & 22,2 & $\begin{array}{c}\mathbf{0 , 9} \\
(1120)\end{array}$ & 40,0 & $\begin{array}{c}1,6 \\
(2849)\end{array}$ & 20,7 & $\begin{array}{c}\mathbf{0 , 9} \\
(1606) \\
\end{array}$ & 31,5 & $\begin{array}{c}1,6 \\
(1951)\end{array}$ & 47,8 & $\left(\frac{2,0}{1881)}\right.$ \\
\hline & 40 & 38,6 & $\begin{array}{c}1,4 \\
(1751)\end{array}$ & 22,3 & $\begin{array}{c}\mathbf{0 , 7} \\
(1079)\end{array}$ & 47,2 & $\frac{1,5}{(1838)}$ & 22,1 & $\begin{array}{c}\mathbf{0 , 7} \\
(1605) \\
\end{array}$ & 37,8 & $\begin{array}{c}1,4 \\
(1819) \\
\end{array}$ & 49,3 & $\frac{1,5}{(2614)}$ \\
\hline & 50 & 43,0 & $\begin{array}{c}1,3 \\
(1701) \\
\end{array}$ & 25,8 & \begin{tabular}{|c|}
$\mathbf{0 , 6}$ \\
$(1433)$
\end{tabular} & 53,3 & $\begin{array}{c}1,3 \\
(1650) \\
\end{array}$ & 23,2 & \begin{tabular}{|c|}
$\mathbf{0 , 6}$ \\
$(1594)$ \\
\end{tabular} & 40,3 & $\begin{array}{c}1,3 \\
(1571) \\
\end{array}$ & 54,6 & $\left(\frac{1,4}{(1257)}\right.$ \\
\hline & 60 & 44,6 & $\begin{array}{c}1,1 \\
(1345) \\
\end{array}$ & 26,2 & $\begin{array}{c}\mathbf{0 , 5} \\
(1039)\end{array}$ & 55,4 & \begin{tabular}{|c|}
1,1 \\
$(1743)$ \\
\end{tabular} & 23,8 & \begin{tabular}{|c|}
$\mathbf{0 , 5}$ \\
$(1275)$ \\
\end{tabular} & 42,2 & $\begin{array}{c}1,1 \\
(1350)\end{array}$ & 56,7 & $\left(\frac{1,2}{(1205)}\right.$ \\
\hline & 70 & 45,3 & $\begin{array}{c}1,0 \\
(1884) \\
\end{array}$ & 26,5 & $\begin{array}{c}\mathbf{0 , 4} \\
(1093) \\
\end{array}$ & 56,6 & $\begin{array}{c}1,0 \\
(1117)\end{array}$ & 24,8 & $\begin{array}{c}0,5 \\
(1033) \\
\end{array}$ & 43,8 & $\begin{array}{c}1,0 \\
(1315) \\
\end{array}$ & 61,0 & $\left(\frac{1,1}{(1531)}\right.$ \\
\hline & 80 & 46,7 & $\begin{array}{c}0,9 \\
(1502)\end{array}$ & 27,9 & \begin{tabular}{|c|}
$\mathbf{0 , 4}$ \\
$(1059)$
\end{tabular} & 60,1 & \begin{tabular}{|c|}
0,9 \\
$(1821)$
\end{tabular} & 24,8 & \begin{tabular}{|c|}
$\mathbf{0 , 4}$ \\
$(1080)$
\end{tabular} & 44,3 & $\begin{array}{c}0,9 \\
(1334)\end{array}$ & 64,1 & $\frac{1,0}{(1898)}$ \\
\hline & 90 & 50,3 & $\begin{array}{c}0,8 \\
(2016)\end{array}$ & 29,5 & $\begin{array}{c}\mathbf{0 , 4} \\
(1003)\end{array}$ & 63,1 & $\begin{array}{c}0,8 \\
(1769)\end{array}$ & 25,7 & \begin{tabular}{|c|}
$\mathbf{0 , 4}$ \\
$(1032)$
\end{tabular} & 45,8 & \begin{tabular}{|c|}
0,8 \\
$(1302)$
\end{tabular} & 64,8 & $\left(\frac{0,9}{1779)}\right.$ \\
\hline
\end{tabular}


Os resultados experimentais consubstanciam as conclusões teóricas advindas da expressão (39), como também dos valores dos limitantes superiores do Desvio Padrão $\mathrm{S}_{\mathrm{DR}}$ apresentados na Tabela 2.

Para ambos os casos quanto aos tempos de preparação das máquinas (independentes ou dependentes da seqüência das tarefas), o grau de equivalência entre os espaços de soluções do problema de programação da produção e do problema cíclico assimétrico do caixeiroviajante tende a aumentar à medida que aumenta o porte do problema quanto ao número de tarefas. Como esperado, observa-se que ocorre um pequeno aumento do Desvio Padrão $\mathrm{S}_{\mathrm{DR}}$ quando se aumenta o número de máquinas. As Tabelas 3 e 4 também mostram que os melhores resultados estão associados às Relações II e IV, enquanto que, na maioria das classes de problemas, os maiores $\mathrm{S}_{\mathrm{DR}}$ ocorreram para a relação VI.

Comparando os resultados da Tabela 3 com os correspondentes resultados da Tabela 4, notase que, de maneira geral, o Desvio Padrão $S_{D R}$ apresenta uma tendência de ser maior quando os tempos de preparação das máquinas são dependentes da seqüência das tarefas. Este fato não é explicado pela expressão (39), que teoricamente é a mesma para os dois casos de tempos de preparação. Uma possível causa de tal tendência está associada ao procedimento de geração da amostra de soluções viáveis. Para tempos de preparação independentes da seqüência das tarefas somente os valores de $\sum_{j=0}^{n-1} X_{[j+1]}^{m}$ e $\sum_{j=0}^{n-1} U B X_{[j+1]}^{m}$ sofrem alteração quando é avaliada uma nova solução (seqüência), enquanto que para tempos de preparação dependentes também ocorre variação na $\sum_{j=0}^{n-1} s_{m[j+1]}$, conforme mostram as expressões (14) e (15).

Conforme já mencionado, o grau de equivalência entre os espaços de soluções do problema de programação da produção e do caixeiro-viajante análogo depende somente da amplitude do Desvio Padrão $\mathrm{S}_{\mathrm{DR}}$. Mesmo assim, convém observar que o desvio relativo médio $\overline{\mathrm{DR}}$ tende a aumentar com o aumento do número de tarefas e do número de máquinas e que os menores valores estão associados às Relações II e IV que forneceram também os menores valores de $\mathrm{S}_{\mathrm{DR}}$.

\section{Considerações Finais}

Neste artigo foi apresentada uma propriedade estrutural do problema de programação da produção flow shop permutacional com tempos de preparação das máquinas separados dos tempos de processamento das tarefas, a qual fornece um limitante superior do tempo de máquina parada entre a sua preparação e o início de execução das tarefas.

Uma aplicação da referida propriedade foi proposta a partir de uma analogia do problema de programação da produção com um problema cíclico assimétrico do caixeiro-viajante. Com o objetivo de avaliar a adequação da analogia proposta foi efetuada uma experimentação computacional sobre uma amostra de problemas. 
De maneira geral, com exceção dos problemas de pequeno porte ( $\mathrm{n}=10$ tarefas), os valores do Desvio Padrão $S_{D R}$ do Desvio Relativo $\operatorname{DR}(\sigma)$ são suficientemente pequenos de forma que a analogia proposta entre os problemas mencionados é adequada e pode ser aceita.

Obviamente, a solução do problema cíclico assimétrico do caixeiro-viajante é uma solução aproximada (heurística) do problema original de programação da produção.

\section{Agradecimentos}

Os autores agradecem as contribuições dos revisores. $\mathrm{O}$ trabalho apresentado neste artigo tem o apoio do Conselho Nacional de Desenvolvimento Científico e Tecnológico - CNPq.

\section{Referências Bibliográficas}

(1) Allahverdi, A. \& Aldowaisan, T. (1998). Job lateness in flowshops with setup and removal times separated. Journal of the Operational Research Society, 49, 1001-1006.

(2) Bagga, P.C. \& Khurana, K. (1986). Two-machine flowshop with separated sequenceindependent setup times: mean completion time criterion. Indian Journal of Management and Systems, 2, 47-57.

(3) Bagchi, T.P.; Gupta, J.N.D. \& Sriskandarajah, C. (2006). A review of TSP based approaches for flowshop scheduling. European Journal of Operational Research, 169, 816-854.

(4) Cao, J. \& Bedworth, D.C. (1992). Flow shop scheduling in serial multi-product processes with transfer and setup times. International Journal of Production Research, 30, 1819-1830.

(5) Cheng, T.C.E.; Gupta, J.N.D. \& Wang, G. (2000). A review of flowshop scheduling research with setup times. Production and Operations Management, 9, 262-282.

(6) Corwin, B.D. \& Esogbue, A.O. (1974). Two-machine flowshop scheduling problems with sequence dependent setup times: a dynamic programming approach. Naval Research Logistics Quarterly, 21, 515-524.

(7) Das, S.R.; Gupta, J.N.D. \& Khumawala, B.M. (1995). A savings index heuristic algorithm for flowshop scheduling with sequence dependent set-up times. Journal of the Operational Research Society, 46, 1365-1373.

(8) French, S. (1982). Sequencing and Scheduling: An Introduction to the Mathematics of the Job-Shop. Ellis Horwood Ltd., West Sussex, England.

(9) Framinan, J.M.; Gupta, J.N.D. \& Leisten, R. (2004). A review and classification of heuristics for permutation flow-shop scheduling with makespan objective. Journal of the Operational Research Society, 55, 1243-1255.

(10) Garey, M.R.; Johnson, D.S. \& Sethi, R. (1976). The complexity of flowshop and jobshop scheduling. Mathematics of Operations Research, 1, 117-129.

(11) Gupta, J.N.D. (1968). Travelling salesman problem - a survey of theoretical developments and applications. Opsearch (India), 5, 181-192. 
(12) Gupta, J.N.D. (1969). Economic aspects of scheduling theory. PhD Dissertation, Texas Tech University, Lubbock, Texas.

(13) Gupta, J.N.D. (1975). A search algorithm for the generalized scheduling problem. Computers \& Operations Research, 2, 83-90.

(14) Gupta, J.N.D. \& Darrow, W.P. (1985). Approximate schedules for the two-machine flow-shop with sequence dependent setup times. Indian Journal of Management and Systems, 1, 6-11.

(15) Gupta, J.N.D. \& Darrow, W.P. (1986). The two-machine sequence dependent flowshop scheduling problem. European Journal of Operational Research, 24, 439-446.

(16) Gupta, J.N.D. \& Stafford Jr., E.F. (2006). Flowshop scheduling research after five decades. European Journal of Operational Research, 169, 699-711.

(17) Gupta, J.N.D.; Strusevich, V.A. \& Zwaneveld, C. (1997). Two-stage no-wait scheduling models with setup and removal times. Computers \& Operations Research, 24, 1025-1031.

(18) Hejazi, S.R. \& Saghafian, S. (2005). Flowshop-scheduling problems with makespan criterion: a review. International Journal of Production Research, 43, 2895-2929.

(19) Johnson, S. (1954). Optimal two- and three-stage production schedules with setup times included. Naval Research Logistics Quarterly, 1, 61-68.

(20) Khurana, K. \& Bagga, P.C. (1985). Scheduling of job-block with deadline in n X 2 flowshop problem with separated setup times. Indian Journal of Pure Applied Mathematics, 16, 213-224.

(21) Moccellin, J.V. (1995). A new heuristic method for the permutation flow shop scheduling problem. Journal of the Operational Research Society, 46, 883-886.

(22) Mood, A.M.; Graybill, F.A. \& Boes, D.C. (1974). Introduction to the Theory of Statistics. McGraw-Hill Kogakusha, Ltd., Tokyo, Japan.

(23) Park, T. \& Steudel, H.J. (1991). Analysis of heuristics for job sequencing in manufacturing flow line work-cells. Computers \& Industrial Engineering, 20, 129-140.

(24) Pinedo, M. (1995). Scheduling: Theory, Algorithms, and Systems. Prentice Hall, Inc., Englewood Cliffs, New Jersey.

(25) Rajendran, C. \& Ziegler, H. (1997). Heuristics for scheduling in a flowshop with setup, processing and removal times separated. Production Planning \& Control, 8, 568-576.

(26) Rios-Mercado, R.Z. \& Bard, J.F. (1996). The flowshop scheduling polyhedron with setup times. Technical Report ORP96-07, Graduate Program in Operations Research, University of Texas at Austin, Austin-TX.

(27) Rios-Mercado, R.Z. \& Bard, J.F. (1998). Heuristics for the flow line problem with setup costs. European Journal of Operational Research, 110, 76-98.

(28) Rios-Mercado, R.Z. \& Bard, J.F. (1999a). A branch-and-bound algorithm for flowshop scheduling with setup times. IEE Transactions, 31, 721-731.

(29) Rios-Mercado, R.Z. \& Bard, J.F. (1999b). An enhanced TSP-based heuristic for makespan minimization in a flowshop with setup times. Journal of Heuristics, 5, 53-70. 
(30) Simons Jr., J.V. (1992). Heuristics in flow shop scheduling with sequence dependent setup times. Omega, 20, 215-225.

(31) Srikar, B.N. \& Ghosh, S. (1986). A MILP model for the n-job, m-stage flowshop, with sequence dependent setup times. International Journal of Production Research, 24, 1459-1472.

(32) Stafford, E.F. \& Tseng, F.T. (1990). On the Srikar-Ghosh MILP model for the N X M SDST flowshop problem. International Journal of Production Research, 28, 1817-1830.

(33) Stinson, J.P. \& Smith, W. (1982). A heuristic programming procedure for sequencing the static flowshop. International Journal of Production Research, 20, 753-764.

(34) Yoshida, T. \& Hitomi, K. (1979). Optimal two-stage production scheduling with setup times separated. AIEE Transactions, 11, 261-263.

(35) Weng, M.X.; Lu, J. \& Ren, H. (2001). Unrelated parallel machine scheduling with setup consideration and a total weighted completion time objective. International Journal of Production Economics, 70, 215-226.

(36) Widmer, M. \& Hertz, A. (1989). A new heuristic method for the flow shop sequencing problem. European Journal of Operational Research, 41, 186-193. 